\title{
COMPUTER SKETCH RECOGNITION
}

\author{
A Thesis \\ presented to \\ the Faculty of California Polytechnic State University \\ San Luis Obispo
}

\author{
In Partial Fulfillment \\ of the Requirements for the Degree \\ Master of Science in Computer Science
}

by

Richie Steigerwald

June 2013 
(C) 2013

Richie Steigerwald

ALL RIGHTS RESERVED 


\section{COMMITTEE MEMBERSHIP}

TITLE: Computer Sketch Recognition

AUTHOR: $\quad$ Richie Steigerwald

DATE SUBMITTED: June 2013

COMMITTEE CHAIR: Zoë Wood, Ph.D. Professor of Computer Science

COMmitTeE MEMBER: Chris Lupo, Ph.D. Professor of Computer Science

COMmitTeE MEMBER: Alexander Dekhtyar, Ph.D. Professor of Computer Science 


\section{Abstract \\ Computer Sketch Recognition}

Richie Steigerwald

Tens of thousands of years ago, humans drew sketches that we can see and identify even today. Sketches are the oldest recorded form of human communication and are still widely used. The universality of sketches supersedes that of culture and language. Despite the universal accessibility of sketches by humans, computers are unable to interpret or even correctly identify the contents of sketches drawn by humans with a practical level of accuracy.

In my thesis, I demonstrate that the accuracy of existing sketch recognition techniques can be improved by optimizing the classification criteria. Current techniques classify a 20,000 sketch crowd-sourced dataset with $56 \%$ accuracy [12]. I classify the same dataset with $52 \%$ accuracy, but identify factors that have the greatest effect on the accuracy.

The ability for computers to identify human sketches would be useful particularly in pictionary-like games and other kinds of human-computer interaction; the concepts from sketch recognition could be extended to other kinds of object recognition. 


\section{Contents}

List of Tables vii

List of Figures viii

1 Introduction $\quad 1$

1.1 Applications For Sketch Recognition . . . . . . . . . . . . . 3

1.2 Application ......................... 4

1.3 Contribution ......................... 5

2 Background $\quad 6$

2.1 Feature Detection . . . . . . . . . . . . . . 7

2.1.1 Edge Detection ................ 7

2.1.2 Corner Detection . . . . . . . . . . . . . . . 8

2.1.3 Other Types of Features . . . . . . . . . . . 9

2.2 Feature Description . . . . . . . . . . . . . . 9

2.2.1 Non-Affine Invariant Feature Descriptors . . . . . . . . . . 11

2.2.2 Affine Invariant Feature Descriptors . . . . . . . . . . . . . 13

2.3 Feature Matching . . . . . . . . . . . . . . . . . . . . . 14

2.3.1 Homography................... 14

2.3.2 Bag of Features . . . . . . . . . . . . 16

3 Prior Work 18

3.1 Sketch Enhancement . . . . . . . . . . . . . . . . . . 18

3.1.1 ShadowDraw ..................... 19

3.2 Sketch Recognition . . . . . . . . . . . . . . . . 20

3.2.1 How Do Humans Sketch Objects? . . . . . . . . . . . . . 21 
4 Sketch Classification Procedure 23

4.1 Bag-of-Features . . . . . . . . . . . . . . . . . . . 24

4.1 .1 Feature Detection . . . . . . . . . . . . . . . . . 26

4.1 .2 Feature Description . . . . . . . . . . . . . . . . 28

4.1 .3 Visual Vocabulary . . . . . . . . . . . . . . . . . . . 35

4.1.4 Feature Vector Generation . . . . . . . . . . . . . . . 38

4.2 Sketch Classification . . . . . . . . . . . . . . . . . . 44

$4.2 .1 \quad$ K-Nearest-Neighbors . . . . . . . . . . . . . . . . . 44

4.2.2 Support Vector Machines . . . . . . . . . . . . . . . . . 45

5 Implementation $\quad 47$

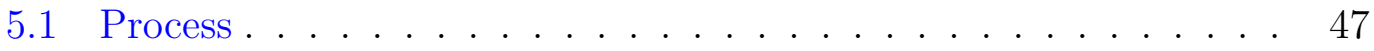

5.2 Local Optimization . . . . . . . . . . . . . . . . . . . 49

5.2.1 Feature Descriptor Computation . . . . . . . . . . . . . 49

$5.2 .2 \quad$ K-means clustering . . . . . . . . . . . . . . . 50

5.2 .3 Soft Feature Vector Assignment . . . . . . . . . . . . 50

5.3 Distributed Approach . . . . . . . . . . . . . . . . . . . 51

5.3 .1 Constraints and Limitations . . . . . . . . . . . . . . 52

6 Results 54

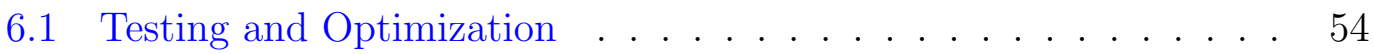

6.2 Feature Detection and Description . . . . . . . . . . . 56

6.3 Bag-of-Features . . . . . . . . . . . . . . . . . 58

6.3 .1 Hard Kernel . . . . . . . . . . . . . . . . . . . . . . . 58

6.3 .2 Soft Kernel . . . . . . . . . . . . . . . . . . . . . . . . . . 59

6.3 .3 Spatial Pyramid . . . . . . . . . . . . . . . . . . 60

6.4 Classification . . . . . . . . . . . . . . . . 61

6.5 Standard Parameters . . . . . . . . . . . . . . . . . . 61

7 Conclusion $\quad 63$

7.1 Limitations . . . . . . . . . . . . . . . . . . . 63

7.2 Future Work . . . . . . . . . . . . . . . . . . . . . 64

8 Bibliography $\quad 66$ 


\section{List of Tables}

6.1 Twenty category subset of taxonomy of 250 categories . . . . . . 55

6.2 Feature Detection and Description Performance Comparison . . . 57

6.3 Hard Kernel Classification Performance . . . . . . . . . . . . . . 59

6.4 Soft Kernel Classification Performance . . . . . . . . . . . . . . . 60

6.5 Spatial Pyramid Classification Performance . . . . . . . . . . 60

6.6 Classification Performance ................ 61 


\section{List of Figures}

1.1 Artists Exaggerate Object Features . . . . . . . . . . . . 3

2.1 Canny Edge Detection Example . . . . . . . . . . . . . . 8

2.2 FAST Corner Detection Example . . . . . . . . . . . . . 9

2.3 SURF Descriptors Example . . . . . . . . . . . . . . . 10

2.4 Calculating SIFT descriptors . . . . . . . . . . . . . . . . . 12

2.5 Homography using SURF and RANSAC . . . . . . . . . 15

2.6 Failed Homography Attempt with Sketches . . . . . . . . . . 16

3.1 ShadowDraw Example . . . . . . . . . . . . . . . . . 20

3.2 How Do Humans Sketch Objects? Taxonomy . . . . . . . . . . . . 21

4.1 Hessian and FAST Examples . . . . . . . . . . . . . . 27

4.2 Two types of feature distributions on images . . . . . . . . 27

4.3 Rotation Invariant Patches Example _ . . . . . . . . . . . . 29

4.4 Gradient Response Bins Example . . . . . . . . . . . . . . 30

4.5 Uniformly and Normally Distributed Patches Example . . . . . 31 
4.6 HOG Patch Example . . . . . . . . . . . . . . . . . . . . . 32

4.7 HOG Patch Sum Example . . . . . . . . . . . . . . . . 32

4.8 HOG Descriptor Visualization . . . . . . . . . . . . . . . . 33

4.9 Feature Descriptor List Example . . . . . . . . . . . . . . . . 34

4.10 Inconsistent Number of Features Example . . . . . . . . . . . . . 35

4.11 Random Feature Descriptors Example . . . . . . . . . . . . . 37

4.12 Visual Vocabulary Visualization . . . . . . . . . . . . . . . . 39

4.13 Similar Descriptors as Different Visual Words Example . . . . . . 40

4.14 Feature Locality Issue Example . . . . . . . . . . . . . . . . . 42

4.15 Spatial Pyramid Representation Example . . . . . . . . . . . . 44

4.16 K-Nearest-Neighbors Example . . . . . . . . . . . . . . . 45

4.17 Two-Dimensional Support Vector Machine Example . . . . . . . . 46 


\section{Chapter 1}

\section{Introduction}

There are millions of things that we do as humans in our day-to-day lives that even with our state-of-the-art knowledge of the brain, we can not come up with a good explanation of precisely how we do those things. Consider a cyclist who rides on a road that she has never seen before but still manages to find her way home. She does not have a built in GPS but her sense of direction does not fail her. Alternatively, consider how a professional baseball player swings his bat to make perfect contact with a ball flying at 100 miles per hour. He is not solving equations in his head to anticipate where the ball will be, yet he can be incredibly precise.

Both of these problems, hitting a baseball and navigating an unknown area, are incredibly complicated in nature. Engineers have come up with good solutions to both of these problems $[28,39]$, ones that could potentially do their jobs better than their human counterparts, but humans are still the only "computers" capable of adequately solving both of these problems and many, many more.

One area of human cognition that engineers including myself are trying to 
emulate is that of vision. For the uninitiated, computer vision is a set of procedures and processes for dealing with data from cameras and other graphical input devices. Generally, it spans everything from digitally reconstructing environments [17] to facial recognition $[43,45]$. However, the field of computer vision is lacking in artwork, specifically in identification, interpretation, and synthesis of art.

Michelangelo once said, "A man paints with his brains and not with his hands." The task of producing artwork is inherently cognitive; as humans we create things where the intent is to communicate some message or emotion to other humans. When we create these messages through visual art, we rely on the receiver to have certain mechanisms to interpret what we drew. In some cases, that mechanism might be the ability to feel the warmth of the colors or to feel the despair of the subject of the painting. However, in many cases the mechanism is simply the ability to identify the subject of the drawing.

Much work has been done in computer vision to try to identify objects in images. Although there is no current way to universally identify all objects in a scene, there are very reliable techniques for identifying objects in photographs. These techniques do not extend very well into the realm of artwork since many objects in works of art tend to not resemble their real-world counterparts. For example, as shown in Fig. 1.1b, when drawing a pig a common stylistic choice is to exaggerate the corkscrew tail.

Because of this, accurately identifying artwork requires knowledge of either the objects the artwork is based on or the stylistic choices made by the artist. This work focuses on identifying drawings based on common stylistic choices. 


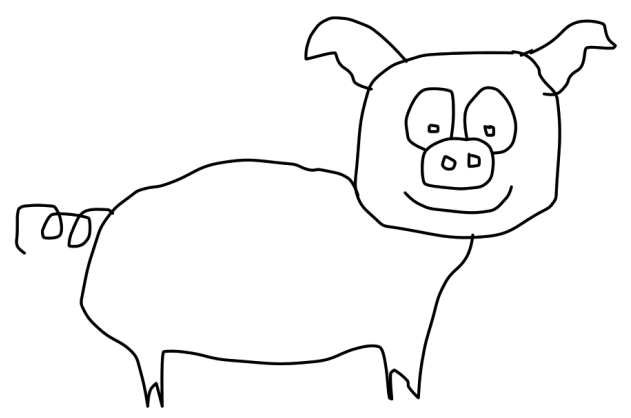

(a) A sketch of a pig with a very curly tail.

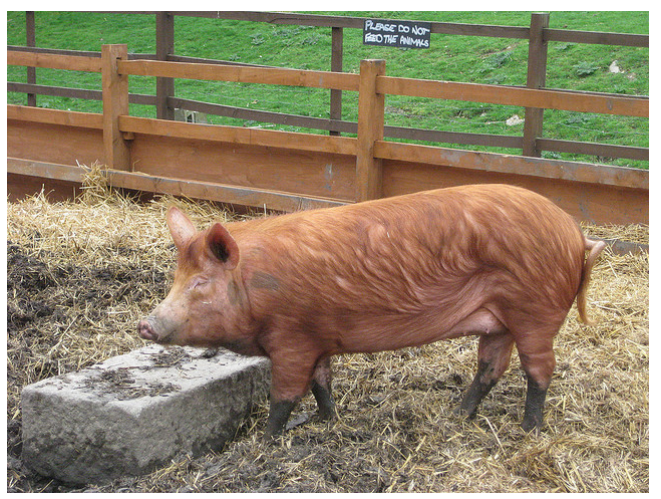

(b) A pig with a somewhat curly tail. Credit: "Jeppestown" (Creative Commons License) .

Figure 1.1: Artists Exaggerate Object Features. Artists tend to exaggerate certain features of objects they depict. This sketch accentuates the eyes, the snout, the mouth, and the tail of the pig whereas in the photo these features are much more subtle.

\subsection{Applications For Sketch Recognition}

Since artwork is made for humans, it might seem unnecessary for a computer to need to interpret it at all, but there are a many reasons that it could be useful to us. Computers could be used for unsupervised cataloging of artwork. Image search engines would be improved greatly if they were able to identify the content of images.

Computers could be used to enhance or restore artwork. There are currently a number of systems that are used to help inexperienced artists create more realistic and identifiable drawings. If the system were able to accurately identify what the user was drawing, it could do a better job of finding similar images or even predicting where other strokes should go for the drawing to resemble the intended object.

Sketch-based systems could be used for games as well. A simple example for a game is a computer teammate or opponent for Pictionary. Another possible 
game could be something similar to Scribblenauts where instead of using text input to generate props, the users could draw the props and the game would be able to predict the user-intended behavior of the drawn prop.

Sketch based systems do not need to be limited to artwork either, a sketch classifier could be used to identify and label components in hand-drafted documents. Many older buildings do not have digital versions of their floor plans, detail drawings, or elevations. Scanning and converting these to digital versions with computer generated labels would facilitate evaluating whether or not older buildings complied to new building codes.

More important than any of these, sketching is one of the most ubiquitous forms of written human communication. Tens of thousands of years ago, humans drew sketches that we can see and identify even today. Sketches are the oldest recorded form of human communication and are still widely used; the universality of sketches supersedes that of culture and language. Because nearly everybody is able to communicate using sketches, in order for humans to better interact with computers, computers must be able to recognize the contents of a sketch.

\subsection{Application}

This work outlines an application that is able to identify the content usercreated line drawings. Users input simple black and white line drawings and the application outputs a category with a level of certainty that the sketch belongs to that category. The classifier is trained with a taxonomy and sample sketches in each category of the taxonomy.

The sketch classifier outlined achieves a similar level of accuracy to prior 
classifiers, but it is designed such that testing can be done incredibly efficiently. Testing a set of parameters on the entire sketch set using a single commodity PC could take over eight hours. With a distributed setup with 100 cores, the same task takes less than five minutes to complete. This enables an efficient search for optimal parameters.

\subsection{Contribution}

One goal of this project was to improve the accuracy of existing sketch recognition techniques. Although the accuracy was not improved, this project demonstrates a technique that could be used to quickly test different image classification solutions on large data sets.

With this technique, a few potential solutions are investigated and the performance and accuracy of each solution is analyzed. Additionally, limitations for each approach are analyzed. 


\section{Chapter 2}

\section{Background}

Object recognition is a broad topic for computer vision and there are many different techniques performed for different applications of object recognition. One current area of focus for robotics and human-computer interaction is gesture recognition [27]. For computer security, biometric analysis (facial recognition, fingerprint recognition, hand print recognition, etc.) is a major area of focus [18]. Research in computer graphics is being done for 3D scene reconstruction from video [17].

Although the processes and algorithms for solving each of these problems vary greatly, there are a few core techniques shared among all of them. One of the most important techniques for computer image processing is the analysis of small distinguishing portions of images called features. A feature is some small piece of an image that may occur in multiple images in multiple places.

Using features in computer vision roughly breaks down into three parts: feature detection, feature description, and feature matching. Feature detection consists of finding locations in images that are semantically significant. Feature 
description consists of converting image patches into symbols that can be easily compared. Feature matching consists of determining a distance metric between two sets of feature descriptions. Modern image classification requires some combination of these three steps.

\subsection{Feature Detection}

There are many approaches for finding distinguishing features in images. Some techniques focus on corner detection, [38] some focus on edge detection, and some involve combined searches [16]. While some approaches are more effective than others for certain tasks [26], there is still no one-feature-type-fits-all.

\subsubsection{Edge Detection}

In computer image processing, edge detection is the process of finding areas in an image where there are sharp changes in levels of brightness. These areas are important because they often correspond to changes in depth of surfaces, changes in orientation of surfaces, and changes in material of surfaces.

Finding edges in images can be an easy task for certain images, like line drawings or fingerprints, where there is a large discrepancy between light areas and dark areas. However, for photographs the separation between objects tends to be blurred which can make edge detection a challenging problem.

The general process of finding edges in the image involves finding zeroes in the second order derivative of the image. There are several variations of this general approach that improve the accuracy for the types of images for which we generally try to find edges $[5,25,31]$. 


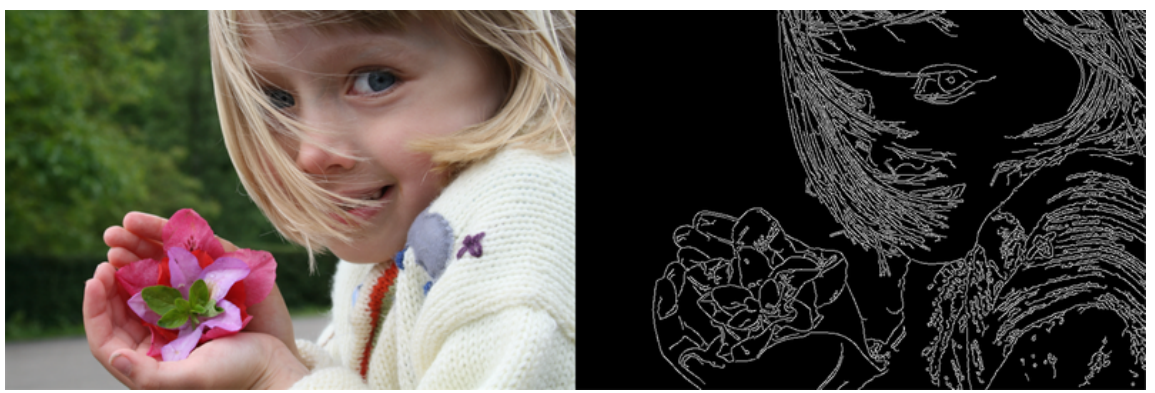

Figure 2.1: Canny Edge Detection Example. Credit: "JonMcLoone" (Creative Commons License)

\subsubsection{Corner Detection}

A corner in an image can be defined as simply as the intersection of two edges. Similar to edge detection however, the problem can be challenging since it is hard to distinguish whether or not certain points actually are corners. In some situations, corners can be rounded or the intersection of edges may blend together and the corner must be inferred from the trajectory of the two edges.

Such challenges make corner detection a very interesting problem. While there have been attempts to solve some of these problems [14], many techniques just ignore these corner cases and simply solve the problem of finding areas where multiple edges have an obvious intersection.

A common approach for finding corners is to find a point in an image where the contrast is high between that point and most of the points surrounding it. One frequently used method that finds corners in this manner is FAST. [34]

For each pixel in an image, FAST creates a circle of sixteen pixels surrounding that pixel with a radius of three pixels. If there are nine pixels in a row in the circle that are either lighter or darker than the center pixel by a threshold, then the center pixel is recognized as a feature. 


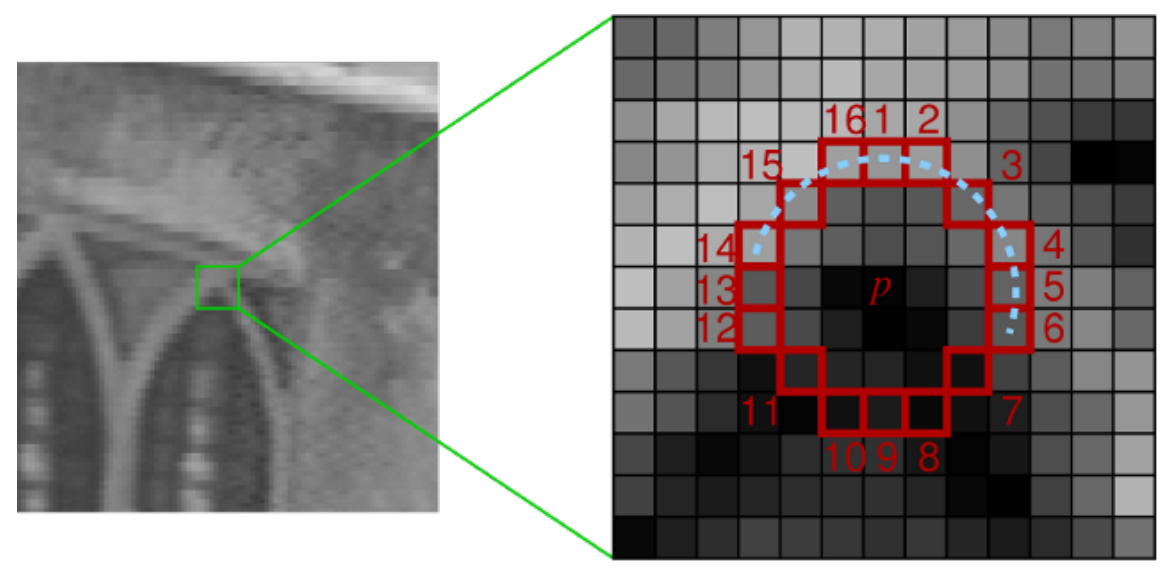

Figure 2.2: FAST Corner Detection Example. (Credit: Rosten and Drummond [34])

\subsubsection{Other Types of Features}

Besides edges and corners, there are several other types of features and shapes that can be distinguished in an image. Two important ones are ridges and blobs. While they may be less frequently used than the other feature detection schemes described, they all serve a purpose in image computation tasks.

\subsection{Feature Description}

Once a feature is found, it is useful to come up with a representation for the features that can be used to compare them with one-another. A representation for a particular feature is often referred to as a feature descriptor.

A number of different types of descriptors for features exist $[2,9,24]$; in practice there are generally two different categories of descriptors, affine invariant and non-affine invariant.

Non-affine invariant feature descriptors are much simpler than affine invariant descriptors. Non-affine invariant feature descriptors only match descriptors for 


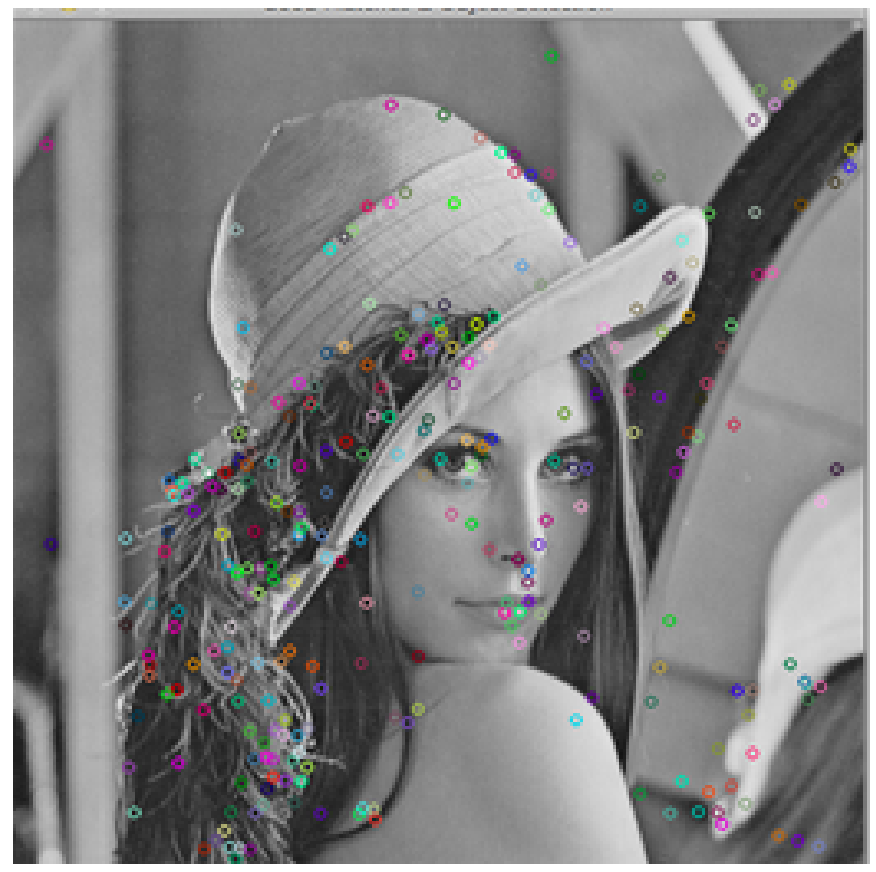

Figure 2.3: SURF Descriptors Example. Lenna image with features identified, and labeled with colors according to SURF descriptors

features that are the same scale and orientation in each image. For a descriptor of a feature in a line drawing, this means that the descriptor encodes the shape, direction, and length of the stroke.

Affine invariant feature descriptors are designed such that they only encode the shape of the feature, but not the scale or the orientation. The benefit of comparing features with affine invariant descriptors is that features discovered in one image are much more likely to match features in another image of the same object from a different angle.

Generally a descriptor is computed by sampling a patch of pixels around a feature point and calculating the sum over $n$ different sub-patches. The feature descriptor is an $n$-dimensional vector where the magnitude of each dimension is the sum of the pixel values of one of the sub-patches. The following sections will go into detail about how descriptors are computed for non-affine invariant 
descriptors and affine-invariant descriptors.

\subsubsection{Non-Affine Invariant Feature Descriptors}

Although affine invariant feature descriptors are more flexible and generally more widely used, several types non-affine invariant descriptors show up quite commonly in recognition tasks. Additionally, affine-invariant features can be looked at as an extension of certain types of non-affine invariant features, so we will look at the non-affine invariant variety first.

One well known type of non-affine invariant feature descriptor is called HoG (Histogram of Oriented Gradients.) It was originally used for pedestrian detection in photographs [9] but it can also be used for classifying the contents of photographs and images [22].

Computing a $\mathrm{HoG}$ feature is simple. The first step involves computing the derivative of the luminosity of the image. This is done to eliminate noise and irrelevant factors in the classification of the image such as relative brightness and exposure.

The next step is locating a point in the image for which to compute a feature descriptor. This can be done with any of the feature detection steps in the previous section, or what is commonly done with HoG features, sampling points uniformly throughout the image.

To generate the descriptor, pixels are sampled in an area around the feature's center called a patch. For photographs, patches are commonly $16 \times 16$ pixels. However, the best patch size for any particular application depends on the scale and the contents of the image. 


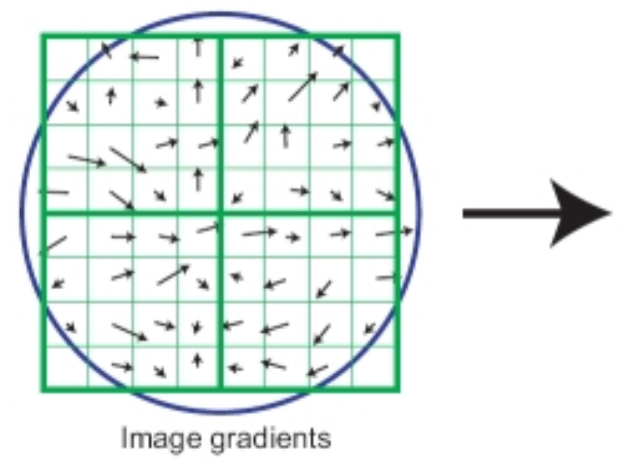

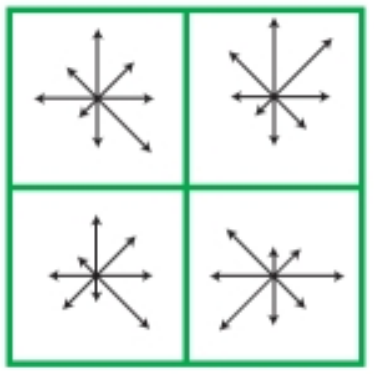

Keypoint descriptor

Figure 2.4: Calculating SIFT descriptors. (Credit: Lowe [24])

Computing the HoG descriptor is almost identical to the initial steps of computing a type of affine-invariant descriptor called SIFT, so Fig. 2.4 is used to aid the explanation of HoG descriptor computation.

In Fig. 2.4 on the left, there is an $8 \times 8$ pixel patch with the value of the image gradient at each pixel indicated by an arrow. The $8 \times 8$ pixel patch is sliced into four $4 \times 4$ pixel sub-patches. For each sub-patch, there are eight bins; each bin represents an angle sampled uniformly from $[0,2 \pi)$. Sometimes the bins are referred to as orientation response bins.

For each pixel in each sub-patch, the magnitude of the gradient vector is added to the orientation response bin for which the angle to the gradient vector is minimized. When the angle of the gradient vector falls between two bins, the magnitude is linearly interpolated between both bins. In the diagram, the sum of the lengths of the arrows in the left image correspond to the lengths of the arrows in the right image.

At this point, there are four sub-patches each with eight orientation response bins. The 32 orientation response bins create the feature descriptor. It can be expressed as a 32-dimensional vector. Most commonly, the $16 \times 16$ pixel patches are broken down into 16 sub-patches each with 8 orientation response bins. This 
creates a 128-dimensional vector.

Non-affine invariant features are actually very fast to compute. This is an important property because images may have many heavily overlapping patches. Computing each response bin can be reduced to a single look-up with the following technique. Convolution of the image with a Sobel or a Scharr kernel sped up with a fast Fourier transform generates the orientation response bins. Then convolution of the orientation response images with a box filter the width of a sub-patch computes the sum of every possible square sub-patch in the image.

Non-affine invariant features are also very fast to compare. For two descriptors $\mathbf{d}_{\mathbf{1}}$ and $\mathbf{d}_{\mathbf{2}}$ their similarity is measured by $\left|\mathbf{d}_{\mathbf{1}}-\mathbf{d}_{\mathbf{2}}\right|_{2}$. If the norm is small, then the features are very similar. Likewise if the norm is large, then the features are different.

HoG feature descriptors are very useful for comparing images where the variation in scale, size, and orientation between images is expected to be very small or non-existent. For this reason, HoG feature descriptors are useful for getting a general impression of an image rather than making a precise judgement about the details of an image.

\subsubsection{Affine Invariant Feature Descriptors}

Affine invariant feature descriptors are very popular for locating similar objects in images and tracking objects in videos because they are invariant to the scales and rotations of the objects. SIFT is one of the original affine invariant descriptors. SIFT stands for scale-invariant feature transform; it is a patented and very widely used process for finding features, representing features, and comparing feature descriptors [24]. 
SIFT is similar to HoG, but able to achieve affine invariance by computing the feature descriptors (as illustrated in Fig. 2.4) by orienting feature patches to face the direction of the consensus of the gradient of the patch where the descriptor is extracted. Additionally, SIFT computes the descriptors at several different scales to achieve scale invariance.

SURF is a popular and commonly used variation of SIFT. SURF, or Speeded Up Robust Features, can be orders of magnitude faster than SIFT, but at the expense of not performing as accurately in certain situations [2]. Along with SURF, there are several other affine invariant feature descriptors for different applications such as ORB (Oriented BRIEF) [36] and FREAK (Fast Retina Keypoint) [1].

\section{$2.3 \quad$ Feature Matching}

Feature matching is the final stage of the image-processing pipeline. Once

we have a set of feature descriptors that describe the content of the image, we can extract a semantic interpretation from those feature descriptors. Two commonly used techniques for comparing sets of features are homography, and bag-of-features.

\subsubsection{Homography}

Homography is very useful for finding pictures of the same object taken at different angles. Consider you have a can of soda and want the computer to be able to identify the brand of soda. By taking a picture of the can and having the computer compare the features from the picture of the can to the Coca-Cola $₫$ 


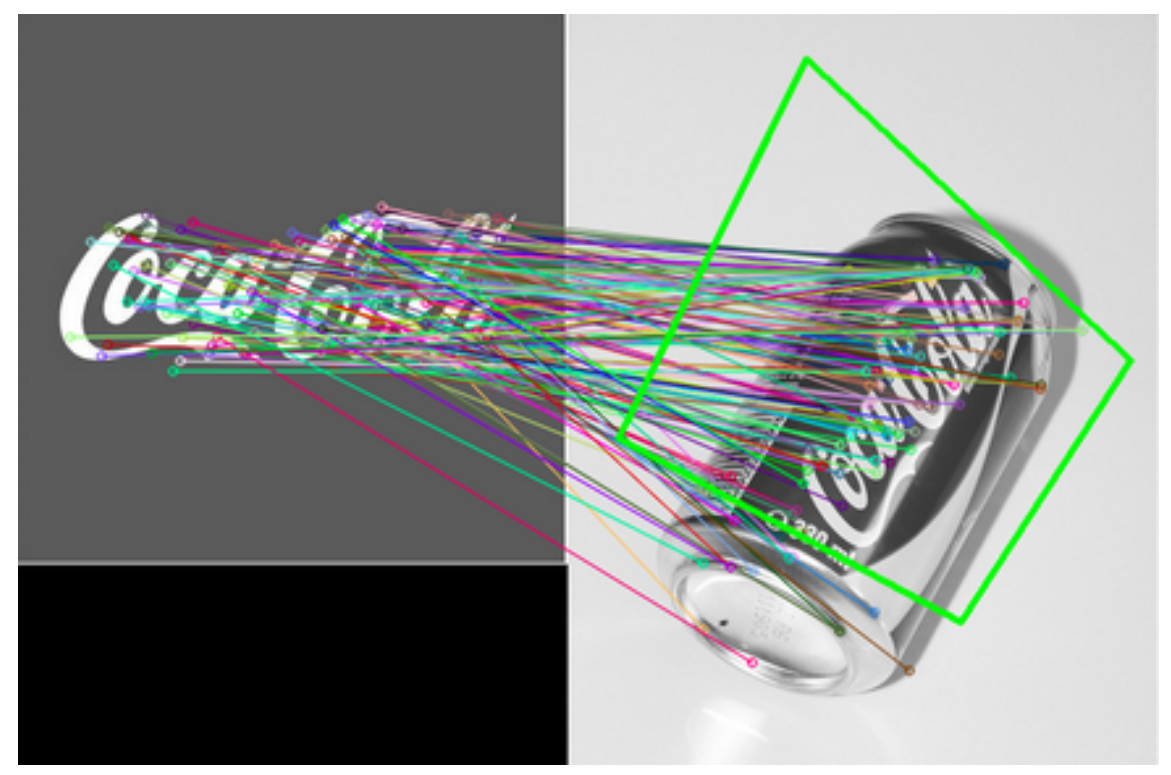

Figure 2.5: Homography using SURF and RANSAC. Homography

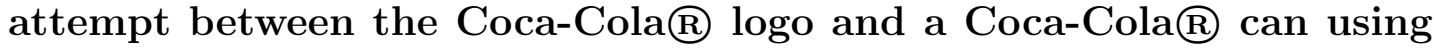
SURF descriptors and RANSAC. Coca-Cola can credit: "byrev" (Creative Commons License)

logo, it can identify that the can has a transformed Coca-Cola ${ }^{\circledR} \operatorname{logo}$ on it. Other brand logos would not be identified on the can with a high level of certainty.

There are several techniques used to perform homography. Two of the most widely used are RANSAC (Random Sample Consensus) and LMedS (Least Median of Squares).

This kind of detection can be done for all kinds of classes of objects; it has been used for video search [19] and even for reconstruction of 3D environments from video [17].

Unfortunately the story is not all unicorns and rainbows. The classification done with the Coca-Cola can requires a transformation from one image space to another, and while that is easy to do in many cases it is only really a solution for the correspondence problem, not for general object comparison. To elaborate, the correspondence problem is the problem of finding what parts of one 


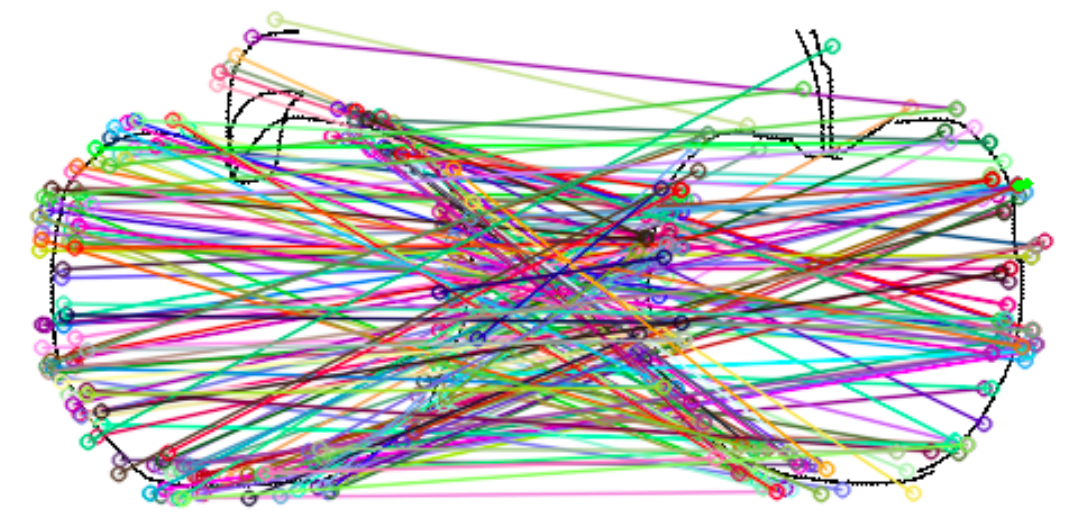

Figure 2.6: Failed Homography Attempt with Sketches

image correspond to some parts of another image. Since homography solves the correspondence problem, it cannot perform a transformation between inherently different objects like from one apple to another similar but different apple as demonstrated in Fig. 2.6.

\subsubsection{Bag of Features}

Bag of features refers to a general process followed for image matching and classification. Two images are similar if the distance between their bag-of-features representations is sufficiently small. The process involves creating an $n$-dimensional vector that represents each image. The $n$-dimensional vectors can be compared with distance metrics, or they can even be classified using common machine learning techniques like support vector machines [8].

This kind of image comparison is used when there may be high levels of variation between similar images. One downside to the bag-of-features approach is that the locations of each feature are not encoded in the final representation of the image. One attempt to remedy this problem involves creating multiple bags of features for different spatial regions in the images [22]. 
Since this project is based heavily on bag-of-features, later sections elaborate much more on this approach to image classification. 


\section{Chapter 3}

\section{Prior Work}

The method described by this paper for sketch recognition and categorization borrows heavily from a number of other current approaches. Most of the research that has been done in the area of computer recognition of line-drawings is in sketch enhancement and object classification.

\subsection{Sketch Enhancement}

Recent approaches to sketch enhancement focus on improving sketches during the process of drawing. The process involves comparing sketched objects to line representations of photographs. The results obtained from these other approaches are due primarily to the sketched objects being geometrically similar to their realworld counterparts $[6,11,41]$.

Instead of learning from other sketches, these applications look at example photographs. Because of this, these applications are only able to recognize sketched objects that resemble their real-world counterparts. Retrieving accurate 
results from poor artists or artists that draw abstractly is difficult because these sketches will not match photographs used for classification.

Sketch assistance systems help create objects that are geometrically similar to real objects. During the drawing process, they search for photos geometrically similar to the sketch. They require either a lot of data, or require text labels for the sketches $[7,10,23]$. One example of such a system is ShadowDraw.

\subsubsection{ShadowDraw}

ShadowDraw is an application that is able to identify a line drawing that an artist creates during the process of drawing. An example of a user drawing a motorcycle using ShadowDraw is shown in Fig. 3.1. While an artist draws, ShadowDraw dynamically suggests additional lines for the artist to draw by showing a "shadow" of blended contours for objects that match the user's strokes. The artist may use the shadows to assist them in creating photo-realistic drawings. It is similar to tracing, but instead of providing a single image, ShadowDraw provides many relevant images to trace from and it updates the suggestions in real-time [23].

ShadowDraw uses the bag-of-features approach outlined in Chapter 2. First, feature points are distributed uniformly across the image, then BiCE descriptors are calculated for each feature point [46]. BiCE descriptors differ from most other descriptors in that they only encode the presence of an edge and the angle, not the magnitude of the gradient of that edge. After the descriptors are calculated, the different types of descriptor are enumerated to generate a histogram for each image. Finding images similar to the sketch is a simple distance comparison between any two histograms. 


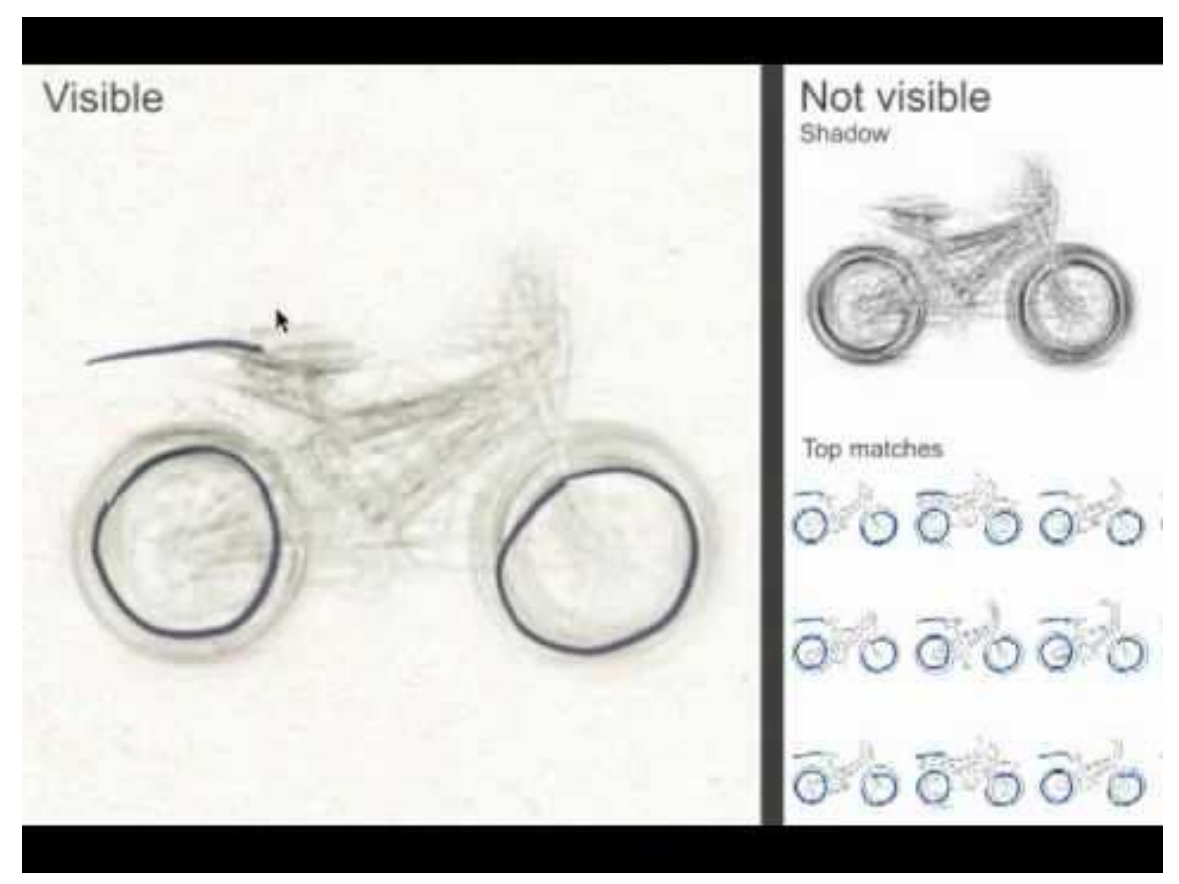

Figure 3.1: ShadowDraw Example. (Credit: [23])

The method used by ShadowDraw could potentially be used for sketch classification. If a sketch matches a particular image, then the class of that image could be used to label the drawing. For example, if the sketch resembles a bicycle, then the closest image matches will be photos of bicycles. Since the categories of the photos are known, they could be used to label the unknown images.

\subsection{Sketch Recognition}

Most previous approaches for sketch recognition focus on domain specific sketches like mathematical equations, chemical diagrams, and handwriting recognition. They build off of approaches that classify different types of individual pen strokes, constructing higher-level diagrams from these lower-level inputs $[15,30,40]$.

Handwriting recognition is one of the most studied forms of computer-pen 

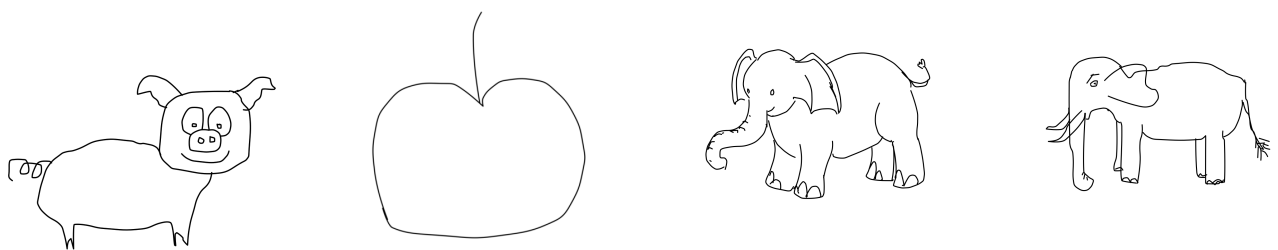

Figure 3.2: How Do Humans Sketch Objects? Taxonomy. A few example sketches in the 20,000 sketch data-set obtained by Eitz et al. $[12]$

input. In the past, gesture-based systems were used for text input, where the order of the strokes and the motion of the stroke (e.g., clockwise versus counterclockwise) were required to specify the shape $[20,35]$. Current techniques for handwriting analysis do not require knowledge of the stroke motion and can be performed on documents that are photographed or scanned [3]. This is ideal since it is likely that most handwriting is not done on computer-tablet interfaces.

Current approaches for sketch recognition in specific application domains are highly structured, like for chemical molecules or for mathematical equations. The known structure of the diagrams can be used to achieve high recognition rates $[21,29]$.

\subsubsection{How Do Humans Sketch Objects?}

For broad categories of drawings, not much research has been done up until recently. Eitz et al. [12] create a dataset of 20,000 human sketches in a taxonomy of 250 object categories using crowdsourcing. The categories include animals like lions and tigers and bears (oh my!) food like apples, carrots, and bananas, and other every day objects like chairs, light-bulbs, and bicycles. Several examples of sketches are shown in Fig. 3.2. The set of 20,000 human sketches is used extensively in this project. 
Along with gathering the image set, they analyze the rate at which humans are able to accurately identify the pictures in the taxonomy to be at least $73 \%$. Additionally, they create a classifier that is able to recognize sketches with an accuracy of $56 \%$. The classifier that they create is very similar to the one used by ShadowDraw, several significant differences are that instead of a nearest neighbor search, they train a support vector machine to train their classifier. Additionally, they incorporate several new techniques for bag-of-features classification into their classifier that enables it to perform with relatively high levels of accuracy. For this project, I analyze their classification method and also develop my own. 


\section{Chapter 4}

\section{Sketch Classification Procedure}

You may have once heard the old expression, "a picture is worth 1000 words." This is a ridiculous claim. But it turns out that the author of the claim, none other than Napoleon Bonaparte, was not too far off though. As it turns out, a picture is actually worth exactly 900 visual words.

Our goal is, given a sketch, to figure out what the subject of the sketch is. In machine-learning, we say that we classify the sketch. The last few decades of machine learning research have provided a number of tools that can be used for classification: Bayes classifiers, nearest-neighbor search, neural networks, decision trees, support vector machines, and many more [44]. In our case, we give the classifier a sketch of a lion, and it outputs "lion" or "tiger", or "bear" (oh my!) but we need to represent the sketch in such a way that that it is very likely to be classified as a lion. In order to determine how to best represent the sketch, it is important to understand how the classifiers actually work.

These classifiers do not actually accept images by themselves as input. Many of them have been generalized to accept their input as points in $n$-dimensional 
space. The classifiers define a set of boundaries in $n$-dimensional space. If the input point falls inside one of those boundaries, we say it is part of that class. The boundaries for that class are determined by using other points in that class $[4,44]$. It is therefore crucial for all of the points in one class to be close to some other number of points in that class.

In order to provide a sketch of a lion to the classifier and to have it actually be classified as a lion, the sketch needs to have a point representation that falls inside the boundary for the "lion" class. In order for the classifier to identify a point representing the image of a lion as being of the class "lion" it must be close in space to the other points representing lions, but separable in space from points representing tigers or bears. To get the most accurate classification, we must use an n-dimensional point representation of an image that segregates points in space that are in different image classes.

\subsection{Bag-of-Features}

The so-called bag-of-features is one good way to convert an image into an $n$ dimensional point. The idea of a bag-of-features representation of an image comes from the text-classification side of machine learning where instead of classifying images, they classify text documents. The particular type of classification that we are mimicking with bag-of-features is called bag-of-words [42].

The idea behind bag-of-words is simple. In order to create a point in $n$ dimensional space for classification, we invent an $n$-dimensional vector space that can be called "interesting word space." The space is build by finding $n$ interesting words, that is, words that show up more frequently in some documents than in others, and use those as basis vectors for the space. A vector representation can be 
constructed for any document by counting the number of times each interesting word appears, and adding the corresponding basis vector for each word to a document vector. To put it simply: count the number of times each interesting word appears in a document to generate a histogram [37].

What text classification researchers found is that texts that humans would classify to be similar tend to have similar numbers of each type of interesting word. For example, articles about baseball would be more likely to have the words "ERA" and "runs", whereas articles about cricket would be more likely to have the words "BBI" and "wickets." That means document vectors for baseball articles have relatively high values in the baseball-related dimensions and the cricket articles have relatively high values in the cricket-related dimensions. This makes it possible to draw a boundary that separates baseball document vectors from cricket document vectors. To classify a new article, simply count the number of interesting words, generate a document vector, and calculate which side of the boundary the document vector falls on.

A similar process can be followed for images. In the image processing domain there is a notion quite similar to a word called a "feature". A feature can be anything like a line, a corner, or some sort of shape that is a small sub-region of a lot of images [38]. Since features in the bag-of-features approach to image classification closely parallel words in the bag-of-words approach to text classification, classes of features are often called "visual words" [42].

In order to classify a set of sketches, it is necessary to figure out what "interesting visual-word space" is. It turns out that when using the bag-of-features approach to image classification that the accuracy depends almost entirely on finding distinguishing features. 
For humans, a distinguishing feature might be a novel hat, a big red nose, or comically large shoes. For the general task of object recognition, although such identifying articles may enable the classification to be performed more accurately, they are not considered distinguishing features. Instead, they are some small pieces of the image that occur more frequently in some classes of images than in others.

\subsubsection{Feature Detection}

There are many established approaches for finding distinguishing features in photographs. Two quite prominent ones, Harris and Hessian feature detectors, are used by a number of image classification techniques. Harris feature detectors find edges and corners in images. Hessian feature detectors find corners and small blobs (a blob is a part of an image where everything is the same or very close in color or brightness; think like the Photoshop paint bucket or magic wand tool.) Another feature detector, FAST (Features from Accelerated Segment Test) is capable of finding corners and small blobs as well. Fig. ?? shows examples of feature points found on an image using two different feature detection algorithms.

Another approach to finding features is to sample them throughout the image [13]. The placement of the uniform samples depends on the desired number of visual words to represent an image, the number of samples per image, and the size of the patch used to calculate the representation. Fig. 4.2 shows two possible ways to distribute features on an image.

The uniform distribution shown in Fig. 4.2a requires no assumptions about the image being sampled, the normal distribution shown in Fig. $4.2 \mathrm{~b}$ assumes that identifying information tends to be closer to the center of an image. 


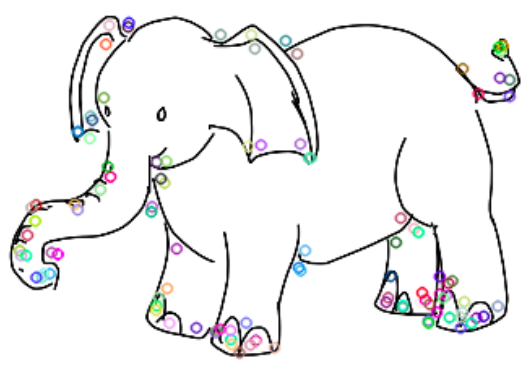

(a) Hessian Features on the much loved elephant

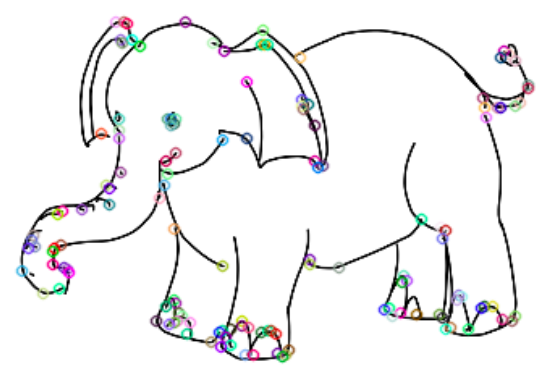

(b) FAST Features on the much loved elephant

Figure 4.1: Hessian and FAST Examples. Two examples of feature detectors used on the sketch of the much loved elephant.

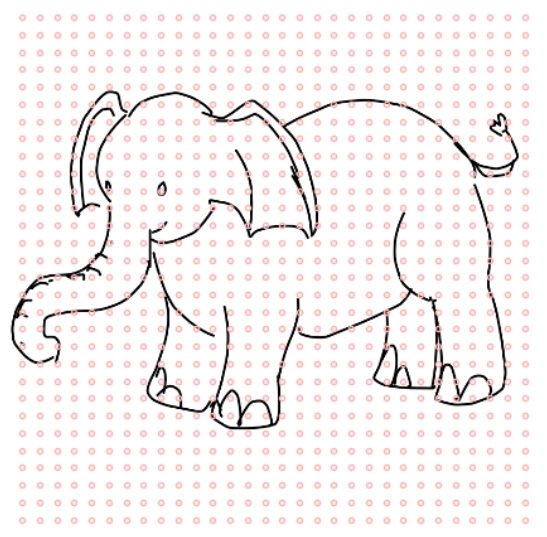

(a) Features distributed uniformly on the much loved elephant

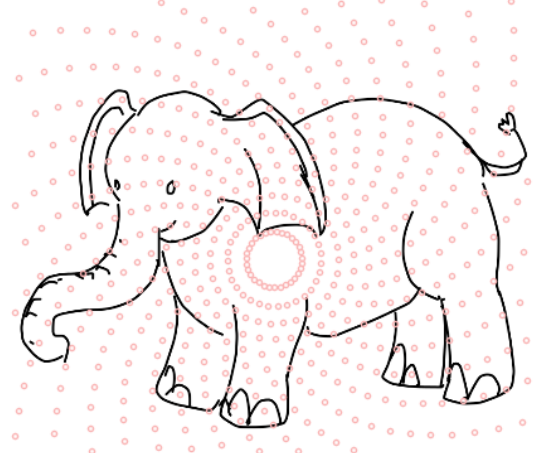

(b) Features distributed normally on the much loved elephant (using boxmuller transform)

Figure 4.2: Two types of feature distributions on images 


\subsubsection{Feature Description}

With a list of feature points, a way to describe those points is needed. In order to determine which visual word each feature could represent, a metric to measure the similarity between two different features is required. The most straightforward approach is to sample pixel values around the point and compare the pixel values for one point to the pixel values for another point. The pixel values around a feature can be represented as a vector, and the distance between two vectors represents the similarity of those features. The most commonly used and most effective feature descriptors do essentially this, but in a way that eliminates noise and other things irrelevant to classification like brightness. Certain other feature descriptors also remove information about the scale and the rotation of the feature.

For the purposes of sketch recognition, eliminating noise is important. Equally important is making the interpretation invariant to stroke thickness and brightness. Preserving the scale and orientation of the features could be either helpful or harmful. If all of the sketches in one category are about the same size and are about the same orientation, then losing information about the scale and the size could actually be detrimental to classification accuracy. Fig. 4.3 shows patches for features containing rabbit's ears and also the stem of a flower. Using a descriptor invariant to orientation, the stem of the flower is classified as the same type of feature as the ear of the rabbit. Using features that encode the orientation would not make the same mistake. However, if the flower stem were instead the ear of an upside-down rabbit it would be better for classifier accuracy if it matched the other two.

Since most of the rabbits in the test set are not hanging from monkey bars and 

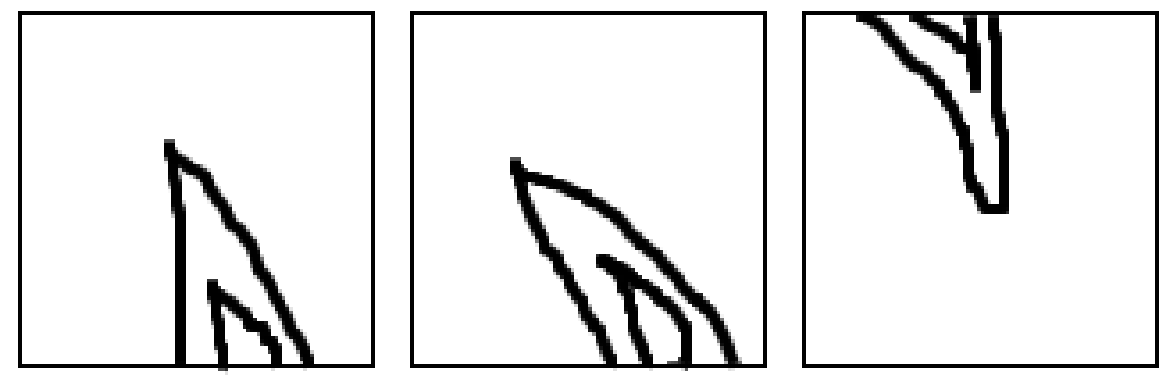

Figure 4.3: Rotation Invariant Patches Example. These two rabbit's ears match the bottom of the flower when using features invariant to rotation.

most other image classes share the same orientation, the accuracy of the classifier should benefit from having the rotation information encoded in the image, but to make sure, both approaches are evaluated.

The type of feature that encodes the orientation in the feature descriptor is called a non-affine-invariant feature descriptor. In the Background chapter an overview for Histogram of Oriented Gradients is provided which is one type of non-affine-invariant feature descriptor. A similar approach for feature representation is used by this application.

To compute a descriptor, first compute the magnitudes of the gradients of the image in several different directions. In Fig. 4.4, four directions are used, 0, $\pi / 4, \pi / 2$, and $3 \pi / 4$. At each point in the gradient, the magnitude is added to the response image whose corresponding angle is closest to the direction at that point, linearly interpolating between images. The four images are called orientation response bins. For this descriptor, only the response for angles between 0 and $\pi$ are used because for line drawings, the response on one side of the line is the inverse of the response on the other side of the line.

Following the creation of the gradient response bins, a descriptor patch is generated for each feature point on the image. A descriptor patch is a slice 

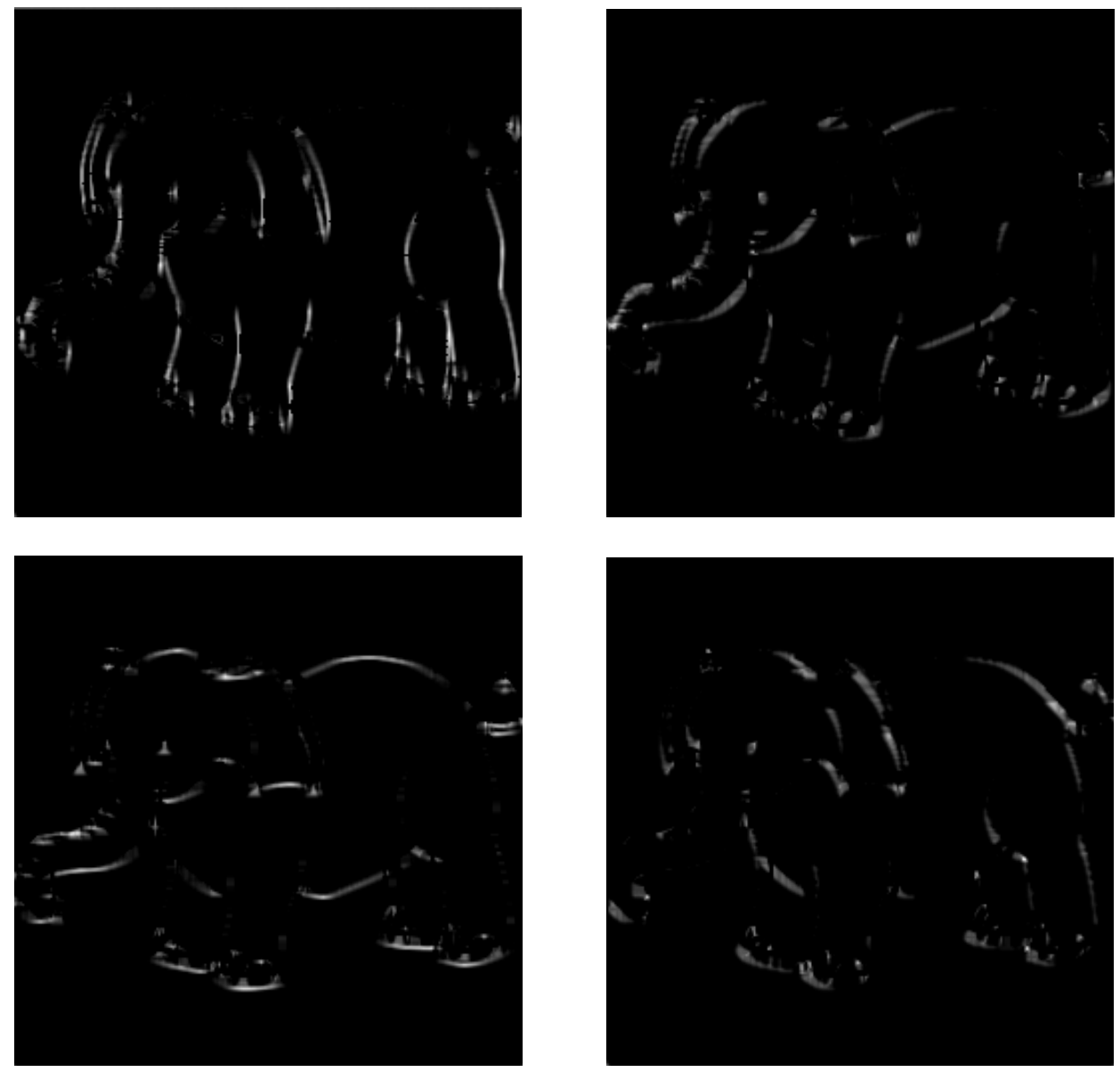

Figure 4.4: Gradient Response Bins Example. Four orientation response bins for the much loved elephant sketch computed for, $0, \pi / 4$, $\pi / 2,3 \pi / 4$ 


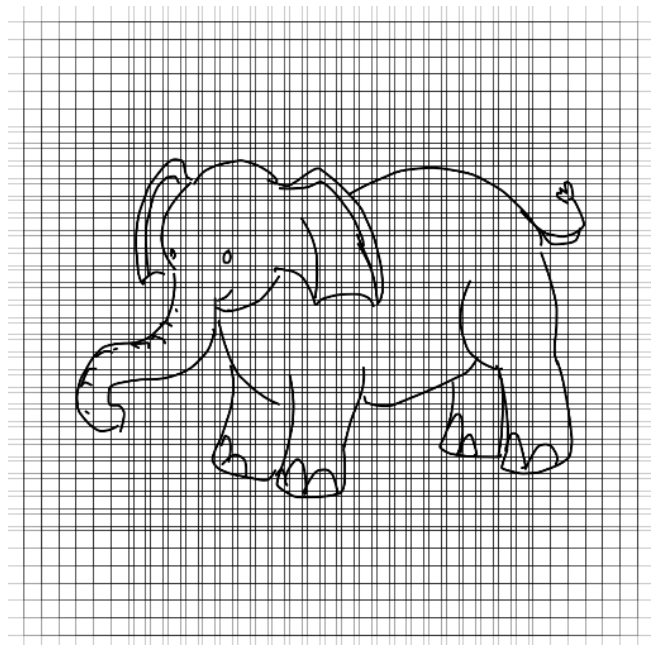

(a) 100x100 pixel Patches distributed uniformly on the much loved elephant

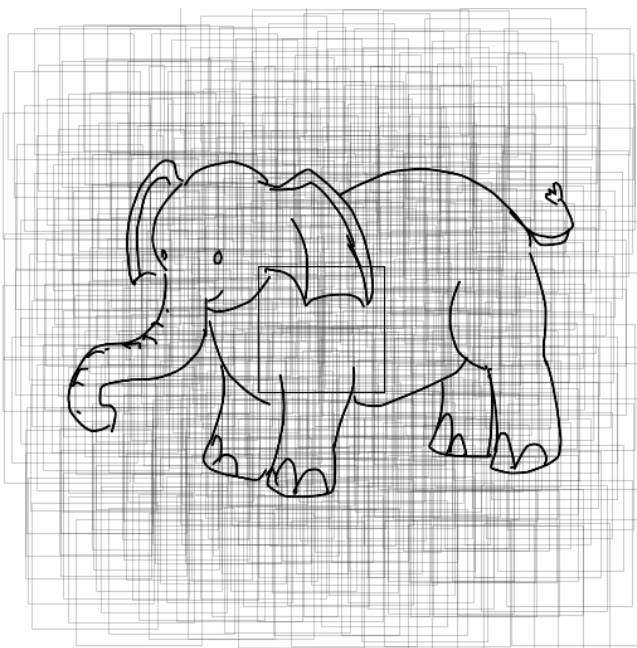

(b) 100x100 pixel Patches distributed normally on the much loved elephant

Figure 4.5: Uniformly and Normally Distributed Patches Example. Two examples of overlapping patch descriptors on the much loved elephant

of the image centered at the feature point. Most modern feature description techniques use square patches, but patches need not be square, for example, fingerprint recognition typically uses round patches [18]. However, square patches are the easiest shape to perform computation on since each patch can be directly represented as a matrix of pixel values.

Since features are very dense on the sketches and the patches for sketches will be relatively large, the patches are heavily overlapping. Fig. 4.5a shows patches on the much loved elephant distributed uniformly and Fig. 4.5b shows patches on the much loved elephant distributed normally about the center.

The square descriptor patch is overlaid on each of the response images. Fig. 4.6 shows an example of a descriptor patch overlaid on the gradient response bins much loved elephant.

Each of the square descriptor patches is subdivided into 16 bins. Each bin 

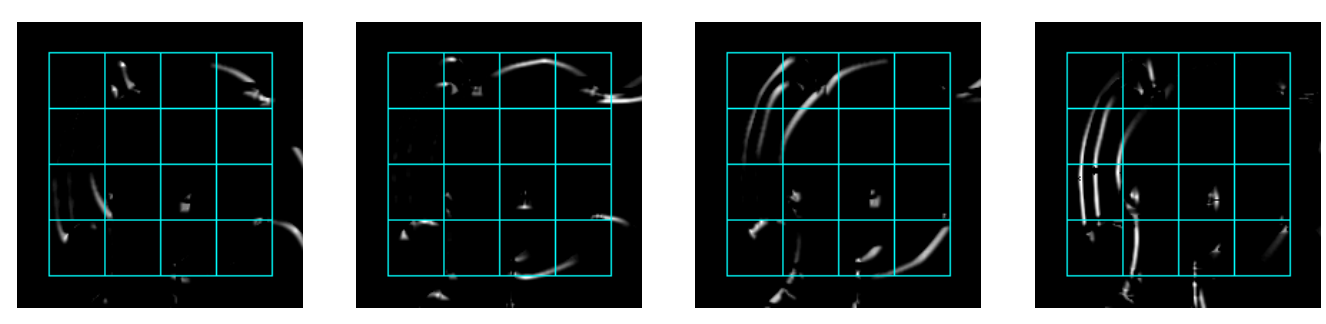

Figure 4.6: HOG Patch Example. Generating bins on the patch descriptor for HOG feature descriptor
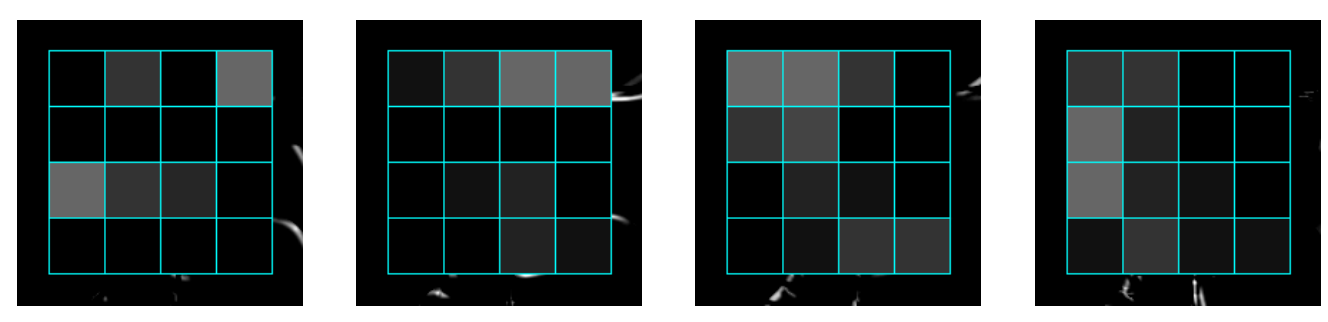

Figure 4.7: HOG Patch Sum Example. Calculate the sum over orientation response.

stores the sum of the portion of the response image contained in that bin. Fig. 4.7 shows an example of the sum over each bin for the much loved elephant.

Once the sum is computed, the vector representation for the feature is constructed by taking the contribution from each bin and adding it to a vector with dimension equal to the total number of bins. A visualization of a descriptor provided by combining the response bins is shown in Fig. 4.8. In the example, there are 64 bins, so a 64 -dimensional vector is created. At this point the descriptor does not resemble the face of the much loved elephant. From this representation of a feature, there is no longer enough information to reconstruct the feature that it represents. The information lost in this process is the thickness of the stroke, the position of the lines within each bin, and positions in the bin where there are variations in curvature.

The lost information in the process of creating the feature descriptor is actually the most important part of the feature descriptor. The goal in creating the 


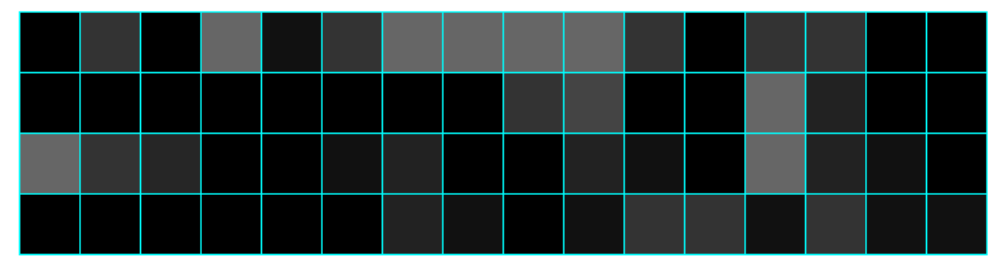

\begin{tabular}{|l|l|l|l|l|l|l|l|l|l|l|l|l|l|l|l|}
\hline 0.0 & 0.3 & 0.0 & 0.5 & 0.0 & 0.3 & 0.5 & 0.5 & 0.5 & 0.5 & 0.3 & 0.0 & 0.3 & 0.3 & 0.0 & 0.0 \\
\hline 0.0 & 0.0 & 0.0 & 0.0 & 0.0 & 0.0 & 0.0 & 0.0 & 0.3 & 0.4 & 0.0 & 0.0 & 0.5 & 0.2 & 0.0 & 0.0 \\
\hline 0.5 & 0.3 & 0.2 & 0.0 & 0.0 & 0.1 & 0.2 & 0.0 & 0.0 & 0.2 & 0.2 & 0.0 & 0.5 & 0.2 & 0.0 & 0.0 \\
\hline 0.0 & 0.0 & 0.0 & 0.0 & 0.0 & 0.0 & 0.2 & 0.1 & 0.0 & 0.1 & 0.3 & 0.3 & 0.0 & 0.3 & 0.0 & 0.0 \\
\hline
\end{tabular}

Figure 4.8: HOG Descriptor Visualization. Combine the bins from each of the response images to create a feature descriptor.

feature descriptor is to selectively get rid of information that we wish to make the descriptor invariant. This way, two descriptors that represent features that are visually similar would have a small linear distance between them. In this case, the saved information is the approximate positions and orientations of lines around the feature. This way, other features that have similar approximate positions and orientations of lines around them will create similar descriptors.

This process is followed for each feature in the image. Fig. 4.9a shows a list of feature descriptors calculated for the much loved elephant where the features are sampled uniformly throughout the image. Fig. $4.9 \mathrm{~b}$ has the same process performed on the apple that looks kind of like a cherry.

It is visually apparent that the two lists of feature descriptors represent different objects. Moreover, the distribution of bin values for the much loved elephant suggest more intricate details throughout the image than that of the apple that kind of looks like a cherry. 

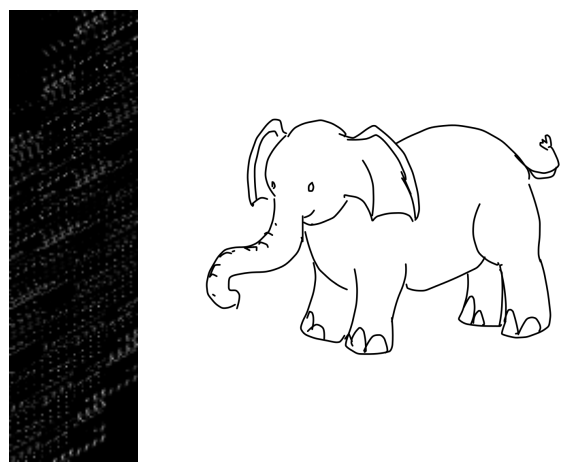

(a) Feature descriptors computed for the much loved elephant
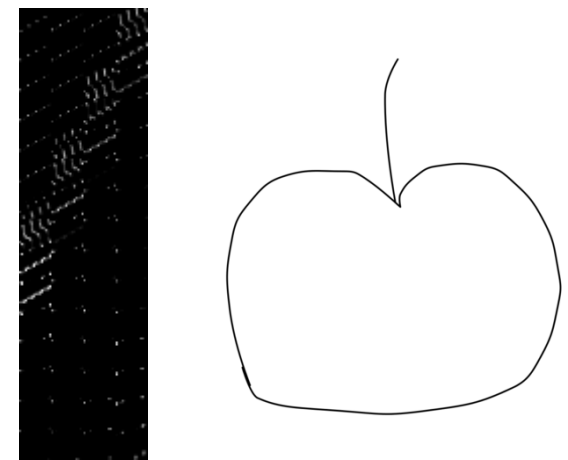

(b) Feature descriptors computed for the apple that looks kind of like a cherry

Figure 4.9: Feature Descriptor List Example. Examples of lists of feature descriptors computed for two different images

These properties of the lists might suggest that a simple distance comparison could be done between the two lists of features. However, there are several problems with comparing the two lists of features so simply. Consider first comparing lists of feature descriptors computed for features found with a corner detection algorithm like FAST. FAST finds 136 features on the much loved elephant, but only finds 8 features in the apple that kind of looks like a cherry. To compare images between classes, the problem is no longer a simple list comparison. Even in classes with the same image list, comparison would be a challenge, for example, FAST finds 97 features on the hopelessly grumpy less loved elephant.

For feature descriptor lists that are the same length like for ones computed by using either the uniformly distributed features or the normally distributed features, a simple comparison could be performed. However, the list directly encodes the exact location of each feature in the image. If there is any variation in position of sketch components, then the lists will contain significant differences. The method of comparison must be invariant to the approximate location of different features within the sketch.

Even if there is an effective way to compare two lists of feature descriptors, a 


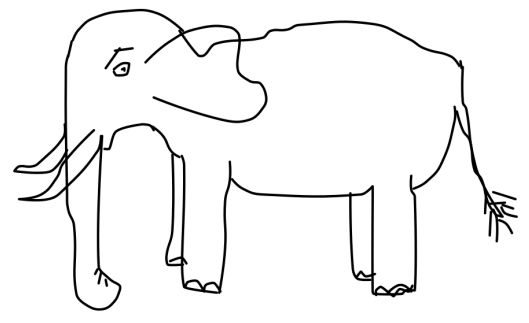

(a) The curmudgeony less loved elephant

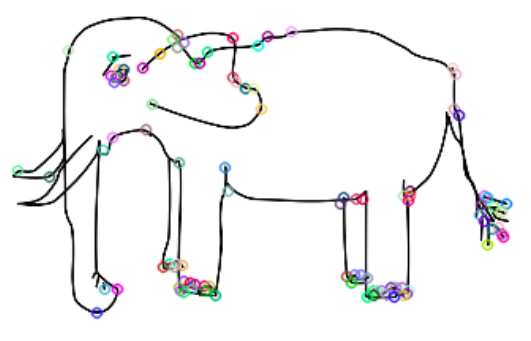

(b) FAST finds 97 features on the less loved elephant. Maybe he is just having a bad day.

Figure 4.10: Inconsistent Number of Features Example. Inconsistent numbers of features can be found within categories.

bigger problem arises: classifying one image requires comparing that image with a bunch of other images. Comparing two lists of points with high dimensionality could potentially be very time consuming and the time required to perform all of the comparisons grows with the number of images needed to compare. An approach for comparison is needed such that the performance is not greatly affected by the number of images compared against, and that makes the comparison invariant to feature locations. The bag of features approach still fits this criteria.

\subsubsection{Visual Vocabulary}

Going back to the analogy of feature descriptors and visual-words, once the descriptors have been computed for the image, the list of descriptors can be thought of as a document of visual words. In order to perform the bag of features approach, the next step is to count the number of times each of the visual words in "interesting visual-word space" occurs in the list and generate a histogram. In 
order to do this, interesting visual-word space needs to actually exist!

In order to create interesting visual word space, we look for feature descriptors that occur more frequently in some classes of images than others. The trouble is, the likelihood that any two feature descriptors will be identical is very small. For most images, each feature descriptor occurs exactly once, and none of the feature descriptors from any image match any of the feature descriptors from any other image. But all hope is not lost! The feature descriptors were designed so they could be compared for similarity with a simple distance metric. So instead, the bag of features approach counts the number of feature descriptors that can be grouped together as a visual word.

Creating a list of visual-words that are "interesting" or "are in some images but not others" is intentionally vague because it is hard to say whether or not a feature descriptor is representative of an image class. Instead, deciding whether or not a visual word is interesting is something that the classifier decides. This makes the task much easier: it is to create a space of visual words that can describe all of the potential feature descriptors. To get an idea of all of the possible feature descriptors that could appear, it is easy enough to sample random descriptors from each of the images. Fig. 4.11 shows 565 randomly sampled descriptors across many different classes of images.

The descriptors are organized to show that there are many that could be considered to be the same visual word. The simplest way to come up with the set of visual words is to take every descriptor in every image, group together ones are similar, and say all of the descriptors in that group belong to that visual word. The set of all of the visual words is called the visual vocabulary. 

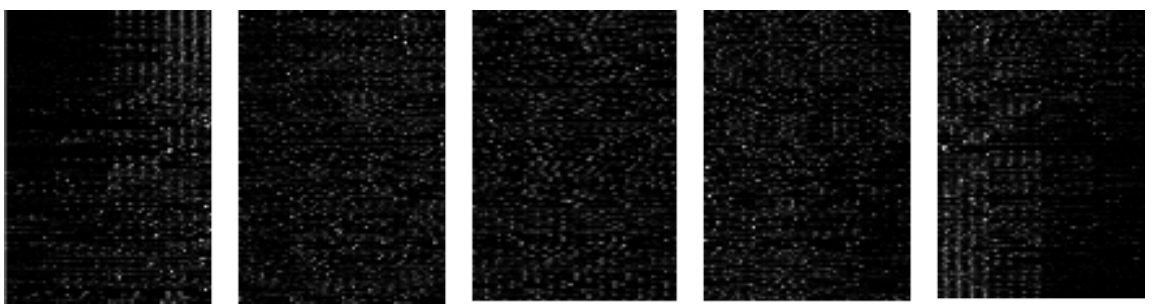

Figure 4.11: Random Feature Descriptors Example. 565 feature descriptors sampled randomly across images, organized by similarity performed by PCA dimensionality reduction and sorting. Each feature descriptor here has 16 position bins and 5 orientation bins for a total of 80 dimensions.

\section{Cluster Analysis}

Grouping descriptors into different visual words is a problem that can be solved with cluster analysis. Many different ways to find clusters in high-dimensional exist, but one of the simplest algorithms, k-means clustering, is sufficient for finding good clusters to build the visual vocabulary.

The goal of k-means clustering, in this case, is given a set of $n$ descriptors, $\left(d_{1}, d_{2}, \ldots, d_{n}\right)$ to find $k$ sets $k \leq n \mathbf{S}=\left\{S_{1}, S_{2}, \ldots, S_{k}\right\}$ where the total sum of the linear distance between each descriptor and the centroid of the set it belongs is minimized. That is:

$$
\underset{\mathbf{S}}{\arg \min } \sum_{i=1}^{k} \sum_{\mathbf{d}_{j} \in S_{i}}\left\|\mathbf{d}_{j}-\boldsymbol{\mu}_{i}\right\|^{2}
$$

where $\boldsymbol{\mu}_{i}$ is the centroid of $S_{i}$ calculated by:

$$
\boldsymbol{\mu}_{i}=\frac{1}{\left|S_{i}\right|} \sum_{\mathbf{d}_{j} \in S_{i}} \mathbf{d}_{j} .
$$

The solution to this problem, $\mathbf{S}=\left\{S_{1}, S_{2}, \ldots, S_{k}\right\}$ is the visual vocabulary. The representation of the visual vocabulary can be simplified by storing only the 
centroids for each set: $\mathbf{M}=\left\{\mu_{1}, \mu_{2}, \ldots, \mu_{k}\right\}$. Each descriptor belongs to the set corresponding to the nearest centroid in $\mathbf{M}$.

Once a visual vocabulary is calculated, different feature descriptors can be classified to different visual words. Fig. 4.12 shows colored patches for the area where each descriptor is calculated on the fanciful castle. Each of the sixteen different colors represents a different visual word. In this example, a dense uniform grid $16 \times 16$ features are sampled where the width of each patch is equal to the distance between each feature. For this example, there are no overlapping patches.

With a visual word for each feature in the image, a bag-of-words representation can finally be computed!

\subsubsection{Feature Vector Generation}

The ever sought-after bag of words representation (or feature vector) of an image is very simple to compute. In summary, each of the sets in the visual vocabulary is labeled to create a basis for the feature vectors, $\mathbf{B}_{S}=\left\{\overline{\mathbf{S}_{\mathbf{1}}}, \overline{\mathbf{S}_{\mathbf{2}}}, \ldots, \overline{\mathbf{S}_{\mathbf{k}}}\right\}$. We can construct a feature vector $\overline{\mathbf{v}} \in\left\{v_{1} \overline{\mathbf{S}_{\mathbf{1}}}+v_{2} \overline{\mathbf{S}_{\mathbf{2}}}+\cdots+v_{k} \overline{\mathbf{S}_{\mathbf{k}}}: v_{1}, \ldots, v_{k} \in \mathbb{R}_{\geq 0}\right\}$ for any image by counting the number of times a descriptor falls in each set of the visual vocabulary, and adding the corresponding basis vector for each visualword to a the feature vector. To put it simply, we count the number of times each visual word appears in the image, and we generate a histogram.

Now this is just one way to do it and although it is a pretty good way, it turns out that it can be improved. The approach described is a pretty standard bagof-words approach, and it has a whole host of shortcomings that can be remedied with slight variations. 


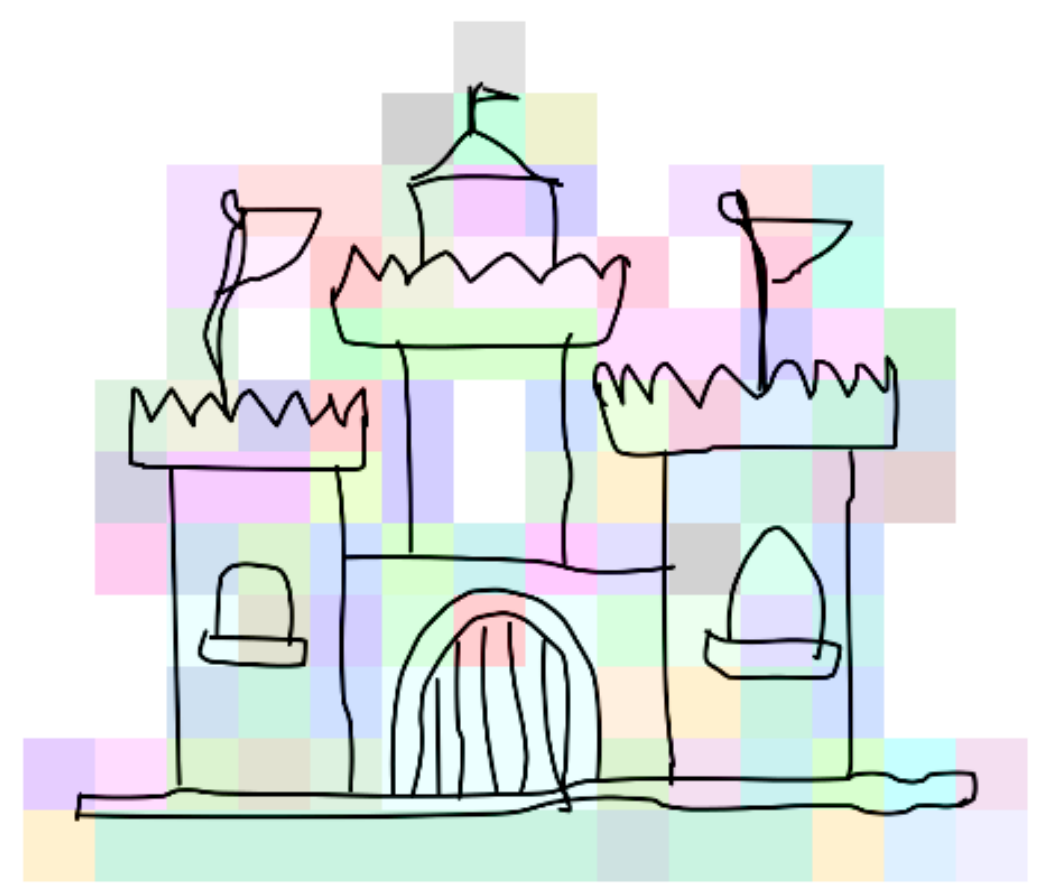

Figure 4.12: Visual Vocabulary Visualization: The fanciful castle; it is a beautiful but impractical place to live. Descriptors are calculated for a dense, non-overlapping grid of patches and colored according to closest visual word centroid. 

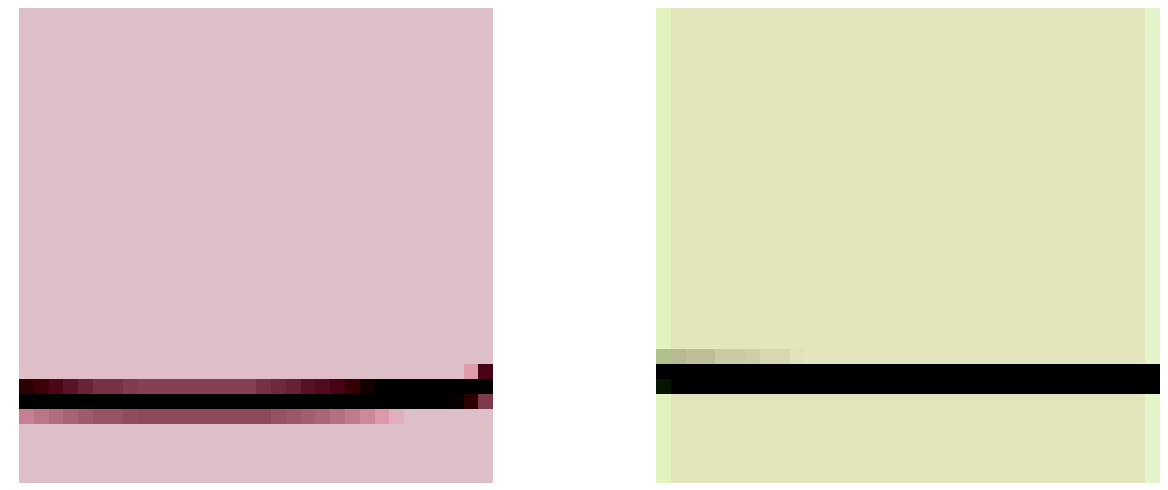

Figure 4.13: Similar Descriptors as Different Visual Words Example. The fanciful castle's separate but equal castle grounds. These are two feature descriptors that represent similar image patches, but are categorized into different words. Now, the puce-colored features / Had patches of puce. / The lemon-chiffon features / Had no such of thuse.

\section{Soft Feature Assignment}

Looking back at a few features on the fanciful castle, Fig. 4.13 shows two patches for descriptors of features each colored according to their closest visual word word centroid along the base of the castle. These two features are very similar and their descriptors are relatively similar as well, however, they each happened to fall under different but similar visual words as indicated by the color.

Suppose there are two very similar but different castle drawings. For one drawing, the foundation is drawn entirely out of lines whose descriptors fall under the same visual-word as the puce-colored feature. For the other castle, the descriptors for the foundation lines fall under the same visual-word as the lemonchiffon-colored features. Even if all else was the same, the two castles would have a distinct discrepancy in the feature vectors even though they look almost identical. This is bad because visually similar images should have equally similar feature vectors in order to be properly classified. Now this is not really the fault 
of the bag-of-words approach, both of the feature descriptors in the example fall very close to the boundary between the two visual words, so the problem really came about because of the k-means clustering. However, these ambiguities can arise with any hard-clustering approach.

There is a way to create feature vectors where these similarities can be accounted for. Instead of assigning each feature to a single set of the visual vocabulary, each feature descriptor contributes to each visual word relative to how far away it is. This method is called soft assignment [32]. In the castle foundation example, each of the two features would contribute about equally to the puce visual word and the lemon-chiffon visual word.

The contribution of each feature to each visual word is calculated by the applying the Gaussian radial basis function to the distance between the feature descriptor and the the centroid of the visual word. Basically, visual words that are close will receive a big contribution and words that are far away will receive a small contribution or no contribution at all. Once the contributions are calculated, the overall contribution of one feature is normalized using the Manhattan norm so that each feature contributes the same amount to the final feature vector as any other feature. Specifically, the feature vector $\mathbf{h}(\mathcal{D}) \in$ $\left\{v_{1} \overline{\mathbf{S}_{\mathbf{1}}}+v_{2} \overline{\mathbf{S}_{\mathbf{2}}}+\cdots+v_{k} \overline{\mathbf{S}_{\mathbf{k}}}: v_{1}, \ldots, v_{k} \in \mathbb{R}_{\geq 0}\right\}$ where $\mathcal{D}$ is the set of descriptors, and $S$ is the visual vocabulary, is computed by:

$$
\mathbf{h}(\mathcal{D})=\frac{1}{|\mathcal{D}|} \sum_{\mathbf{d}_{j} \in \mathcal{D}} \frac{\mathbf{q}\left(\mathbf{d}_{j}\right)}{\left\|\mathbf{q}\left(\mathbf{d}_{j}\right)\right\|_{1}}
$$

where $\mathbf{q}(\mathbf{d})$ is a function that computes the contribution for the descriptor $\mathbf{d}$ 


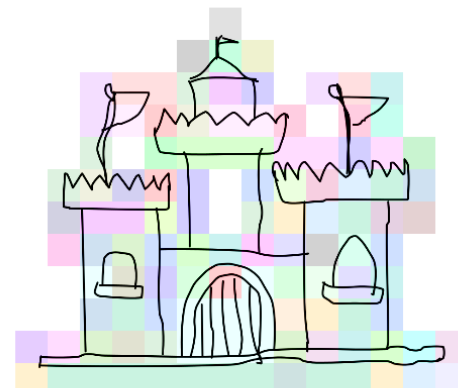

(a) Original Castle

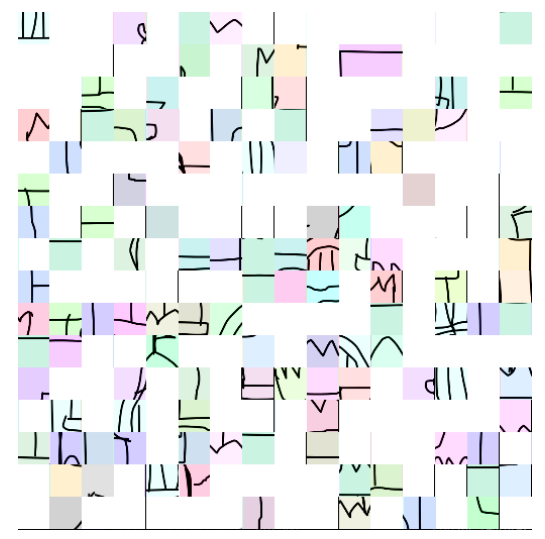

(b) Rearranged Castle

Figure 4.14: Feature Locality Issue Example. These images would match when just comparing the count of each feature.

using the visual vocabulary $S$ defined by:

$$
\mathbf{q}(\mathbf{d})=v_{1} \overline{\mathbf{S}_{\mathbf{1}}}+v_{2} \overline{\mathbf{S}_{\mathbf{2}}}+\cdots+v_{k} \overline{\mathbf{S}_{\mathbf{k}}}: v_{i}=e^{-\left\|\mu_{i}-\mathbf{d}\right\|^{2} / 2 \sigma^{2}}
$$

Where $\sigma$ is a constant that defines the standard deviation for the radial basis function.

This method of calculating the feature vectors eliminates issues for visually similar features that fall on boundaries.

\section{Spatial Pyramid Representation}

One other issue with the bag of features approach is that the locations of the features are lost. Again, going back to the example of the fanciful castle, we could completely rearrange all of the features in the image and it would still match the original castle as shown in Fig. 4.14.

Of course Fig. 4.14 depicts an extreme example, most of the time the descriptor patches are overlapping, which would make it complicated to generate two 
different arrangements with the same descriptors. However, with the overlapping descriptors the problem could still occur.

Potentially the classification could be improved by accounting for the relative positions of different features. We have already looked at one way of comparing images based on feature location; in Chapter 2 we saw that homography is used frequently to compare features in photographs but performs poorly on sketches.

Bag-of-features performs surprisingly well on sketches without information about the relative positions of features, but the performance could be improved by encoding some information about the locality of features. One way of comparing images with bag-of-features representations while preserving positional information is called spatial pyramid matching.

The way spatial pyramid matching works is quite simple. Along with computing the normal bag of features representation for the whole image, we subdivide the image into quadrants and we compute the bag-of-words histogram for each quadrant. This gives us 5 different bag of features representations, one for the whole image and one for each quadrant. Creating a single bag-of-features from the five representations is as simple as assigning a weight to each histogram and concatenating them together. Figure 4.15 shows a toy example of spatial pyramid being performed on a set of features two levels deep. In the example, each histogram is weighted according to the pyramid match kernel outlined in Lazebnik et al. [22]. 

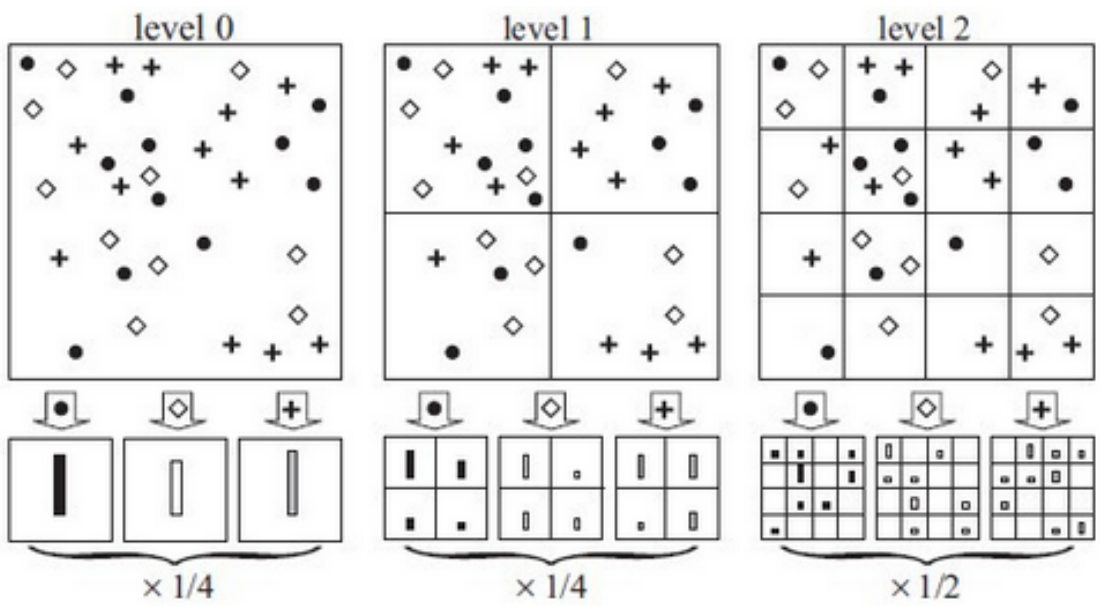

Figure 4.15: Spatial Pyramid Representation Example Lazebnik et al. $[22]$

\subsection{Sketch Classification}

In this section, we will take a look at a number of ways to compare the feature vectors that were generated by the bag-of-features process.

\subsubsection{K-Nearest-Neighbors}

The nearest-neighbor approach (illustrated in Fig. 4.16) is very simple and serves as a baseline for the other classification techniques. For each image to classify, compare the distances between the feature vector for that image and the feature vectors for all images in the training set. The predicted class is the class of the image with the shortest distance.

$\mathrm{K}$ nearest neighbors classification is performed by searching for the $k$ closest neighbors, and picking the class based on the most frequent class of those neighbors. This technique serves as a baseline for other classification techniques. 


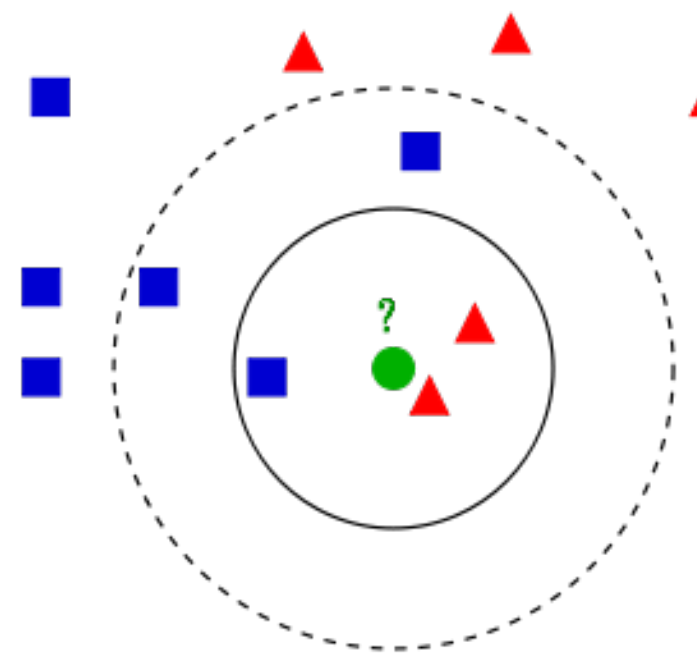

\section{Figure 4.16: K-Nearest Neighbors Example (Creative Commons Attribution-Share Alike. Credit: Antti Ajanki)}

\subsubsection{Support Vector Machines}

Support Vector Machines are a very popular and commonly used type of classifier. SVMs typically classify data into two partitions, but multiple SVMs can be used for multi-class classification. SVMs work by partitioning $n$-dimensional space with boundaries defined according to "support vectors" and a kernel function. The proximity of a point to the boundary is related to the certainty that the object being classified is part of the class designated. A two-dimensional SVM is illustrated in Fig. 4.17.

Support Vector Machines have explicit training and classification steps. That is, a Support Vector Machine is trained with a set of data, and the SVM remains unchanged while it performs predictions about other data.

Since SVMs are inherently two-class classifiers, training the SVM requires actually training different SVMs for each class. Each SVM decides if it is "part of that class" or "not part of that class". That is, each SVM is trained with two classes, one class contains all of the vectors corresponding to images in that class, 


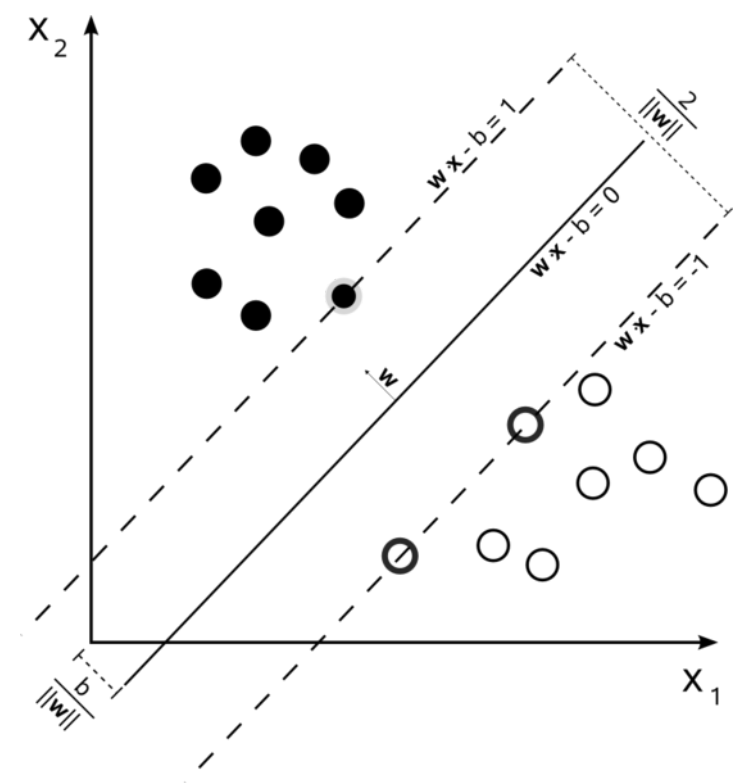

Figure 4.17: Two-Dimensional Support Vector Machine Example (Credit: "Cyc")

the other class contains all of the vectors not corresponding to images in that class. 


\section{Chapter 5}

\section{Implementation}

Although there are many more potential improvements that could be made, investigating the performance of these various features provides insight about which adjustments provide the greatest accuracy.

Implementations of a few variations of the processes outlined in Chapter 4 were done in a combination of $\mathrm{c}++$ and NVidia CUDA. This project relied heavily on OpenCV for image processing and machine learning tasks, on Boost for many

$\mathrm{c}++$ features, and on MPI (using the MPICH implementation) for performing the distributed computation.

\subsection{Process}

The program is broken down into two training steps and one evaluation step. This section contains implementation details for each of these steps, many of which may affect the overall accuracy of the classifier.

The first classification step performed is calculating the centroids for the visual 
words. In order to calculate the centroids, we need all, or a subset of all of the visual words. One by one, the program calculates the descriptors for each image by doing the following.

First, the program loads a grayscale image and crops it to a square according to the longest side of the contained sketch. The image is then scaled to $256 \times 256$ pixels. Once the image is scaled, features are detected using one of the many feature detection algorithms outlined above. For the uniform and normal distributions, features are located only in areas where a descriptor patch fits entirely on the image, so for a 100 pixel wide descriptor patch, no features would be found within 50 pixels of the edge of the image. Once the features have been detected, the image and the feature locations are passed to a feature descriptor extractor such as HoG, SIFT, SURF, FREAK, etc. The extractor calculates descriptors for every feature found in the image. With a list of all of the descriptors from every image, we perform k-means clustering on the descriptors to find the centroids for the visual vocabulary. The descriptors for each image are cached, and the visual vocabulary centroids are saved.

The saved visual vocabulary centroids and the descriptors for each image are both used by the next step of the classification, training the classifier. Using the descriptors, a bag of features representation is generated for each image. For each descriptor in an image, the nearest visual vocabulary centroid is located and the visual word corresponding to that centroid is added to the bag-of-words. In this step, soft kernel coding and spatial pyramid may be performed as well. Once the bag-of-words feature vector has been obtained for each image, we train an SVM with all of the feature vectors. The SVM is used to classify new images.

The last step, the evaluation step, is used to classify images not part of the training set. The images are loaded, converted to grayscale, and cropped. 
Features and descriptors are computed using the same method as used for images in the training set. The same process is also followed to create the bag-of-words representation as for the training set, using the centroids obtained from the first training step. Once the bag-of-words feature vector is obtained for an image, it is classified by the SVM.

\subsection{Local Optimization}

All of the tests run were on the database of 20,000 images provided by Eitz et al. [12]. Initially, there were a few operations that took a long time to process. This was problematic because the data that took the longest to create also changed for each test so it did not make sense to cache intermediate results between tests. Since these operations would be the bottleneck in a standalone or a distributed setup, they needed to be fast so a multitude of tests could be run efficiently.

\subsubsection{Feature Descriptor Computation}

Feature descriptors require a sum over a portion of an image. In some cases, the patches are heavily overlapping. In some experiments there were pixels that were covered by over 900 different patches. For the experiments that performed relatively well, each pixel is still sampled for an average 56 different descriptors. In order to prevent having to sample every pixel in the image 56 times, there is a way to optimize the image so calculating the patch for a descriptor can be done with a single pixel look-up.

From the image, we calculate the gradient by performing a convolution with 
two Scharr kernels. From this we get two images, one that has the magnitude of the gradient along the $\mathrm{x}$ axis and one for the $\mathrm{y}$ axis. The angle of the response at each pixel is calculated by taking the arc-tangent of the quotient of the respective pixels in each image. The angle determines the two response images to which the magnitude contributes.

Once the orientation response bins are calculated, the sum of an every possible sub-patch bin can be calculated by convolving the image with a box filter that has a kernel the same size as the dimensions of the bin. The box filter is sped up using a Fast Fourier Transform. This way, the sum of a response bin becomes a single pixel look-up.

\subsubsection{K-means clustering}

Solving the k-means problem is actually NP-Hard, but Lloyd's algorithm can be used to approximate a solution. The implementation I use, which is part of OpenCV, is single threaded, and thus becomes a computational bottleneck for the whole image recognition process. Computing cluster centers for the whole data set of 20,000 images can take several days in some circumstances. So instead of clustering all feature descriptors from all images, we cluster a random subset of those descriptors. In my experiments, I found that a subset of 100,000 descriptors provided reasonably accurate results while only taking a couple of minutes to find the cluster centers.

\subsubsection{Soft Feature Vector Assignment}

Feature vector assignment is the step where we generate a histogram from a set of feature descriptors coming from a single image. The hard feature vector 
assignment is easy, it is just the nearest-neighbor problem which is easy to solve using $\mathrm{k}$-d trees. However, with soft assignment, a feature may contribute to a single visual word, it might contribute to a few, or it might even contribute to all of them. Because of this, it is necessary to calculate the distance between all descriptors in an image and all centroids in the visual vocabulary.

So there are $|\mathbf{S}| \times|\mathcal{D}| \times \operatorname{dim}(\mathbf{d})$ operations, where $\mathbf{S}$ is the visual vocabulary, $\mathcal{D}$ is the set of feature descriptors for an image, and $\mathbf{d}$ is a feature descriptor in $\mathcal{D}$. Each of these operations could be performed independent of one-another. In a typical situation, $|\mathbf{S}|=500,|\mathcal{D}|=961$, and $\operatorname{dim}(\mathbf{d})=64$. For this case, there are $30,752,000$ floating point operations that could theoretically be done in parallel.

On a single core, this can not be done in real-time with current hardware. The CPU implementation takes an average 0.41 seconds to run for each image on the Intel Core 2 lab machines in room 235. An identical process implemented in CUDA $\mathrm{C}$ to be run on the GPU calculates soft-kernel feature vectors 142 times faster at an average of 2.9 milliseconds per image.

\subsection{Distributed Approach}

Since it took a long time to classify the images on one computer (with the entire image set it took around 8 hours), I decided to run the algorithm on multiple computers at the same time. The Cal Poly computer science labs combined have at least 243 computers (with over 800 cores) that I was able to connect to. At least 47 of them have NVidia graphics cards and are able to run the CUDA implementation of the soft-kernel computation.

I compiled OpenCV, a few Boost libraries, and MPICH for each of the different 
architectures in the different labs so that I could run it on all of them at the same time.

The problem I was trying to solve was what are the best parameters to find features, describe features, enumerate features, and classify the feature vectors. To solve this problem, I could test different parameters on different computers, or I could run the same parameters on a bunch of computers and have each computer perform a piece of the computation. Due to the constraints for my setup, I decided to do the latter.

I determined as a baseline that if the algorithm was perfectly parallelized, that it would take just over 30 seconds to run. There were a number of limitations that prevented me from achieving 30 seconds, but I was able to run the tests in much shorter time than 8 hours.

I had a master delegate tasks to each of the slaves. Each task was an individual image to categorize. The master would send images out to each slave, each slave would compute all of the descriptors for each image and send a sample of random descriptors back to the master. The master would then perform k-means clustering on the descriptors and send the cluster centers to each of the slaves. The slave computes the feature vectors for each of its images and sends the feature vectors to the master.

The master then sends all of the feature vectors to each slave. Each slave then trains and tests support vector machines from different subsets of the data.

\subsubsection{Constraints and Limitations}

Even though the distributed setup was much faster than before, there were a few things that prevented me from achieving my predicted optimal speedup. 
The SVM and the k-means clustering are the bottleneck for this approach since scaling the cluster does not speed up either of these two parts. One potential way to improve this system would be to use Mahout or another distributed machine learning approach. Unfortunately, the Cal Poly lab environment was unfriendly for this kind of setup.

Another problem encountered from this setup was issues caused by unreliable file access and message passing. Due to the nature of the system, data cached on the disk was frequently corrupted. All data cached on disk needed to be read back in and checked after it was written to ensure accuracy. This added significant overhead since the caches were often over 100 megabytes. 


\section{Chapter 6}

\section{Results}

One of the primary goals of this project was to evaluate different modern methods for sketch classification, and to determine which strategies are the most effective for obtaining high classification accuracy. Additionally, since sketching would primarily be performed on tablet interfaces, which generally are less computationally powerful, performance is very important. A number of tests were run that evaluated the accuracy of classification and performance using different types of feature detectors and descriptors. Additionally the tested methods of classification that are most accurate and least time-consuming are identified.

\subsection{Testing and Optimization}

Most of the tests run are on a subset of 20 of the 250 categories. The categories chosen for classification are in table 6.1. The classification results for the subset of twenty classes is better than the classification for the 250 categories, but the results could be demonstrated to be very similar for 250 categories. 
For each run, cross-validation is performed using all 80 images in each category. For training and testing, the 1600 total images are split up into 40 folds of 40 images, each fold contains two images of each class. Forty SVMs are trained, one to test each of the folds. The SVMs are trained with the 39 remaining folds. This way, each image can be tested against nearly the rest of the entire image set.

$\begin{array}{cccc}\text { seagull } & \text { panda } & \text { armchair } & \text { tire } \\ \text { ashtray } & \text { snowboard } & \text { feather } & \text { pigeon } \\ \text { suv } & \text { pickup truck } & \text { barn } & \text { bush } \\ \text { cup } & \text { race car } & \text { van } & \text { streetlight } \\ \text { teacup } & \text { trombone } & \text { parachute } & \text { bottle opener }\end{array}$

Table 6.1: Twenty category subset of taxonomy of 250 categories

Additionally, a two-class test is performed as a baseline where the separation between the classes should be unambiguous. The classes used for the two-class classification are "barn" and "cup".

The goal of testing was to find the strategies and the sets of parameters for each strategy that provided the highest level of classification accuracy. For feature detection, the variables changed are:

- The number of features per image

- The detection type (uniform, normal, FAST, Hessian)

For feature description, the variables changed are:

- The descriptor type (SIFT, FREAK, ORB, HoG)

- The patch size

- The number of bins per patch 
- The number of directions per patch

For bag-of-features construction, the variables changed are:

- The descriptor assignment type (hard/soft kernel)

- Whether or not a spatial pyramid representation is used

For classification, the variables changed are:

- The classifier type (SVM, K nearest neighbors)

- For SVM, the kernel type (RBF, linear)

- The SVM kernel parameters

Unless explicitly stated otherwise, for each of the following tests the following parameters are used:

- $30 \times 30$ grid of uniformly distributed features

- HoG-based descriptors with 4 orientation bins and $4 \times 4$ spatial bins sampled from a $92 \times 92$ pixel patch.

- Soft feature assignment with a two-level spatial pyramid representation

- Linear SVM kernel

\subsection{Feature Detection and Description}

Three different combined feature detection and description schemes were tested: 
- Uniformly distributed features with HoG descriptors

- FAST features with FREAK descriptors

- SURF features and descriptors

The number of features detected by each of the last two depends on the content of the image. FAST and SURF both have thresholds for sensitivity when detecting features. For all of the tests using FAST and SURF detection, the threshold is set so the average number of features detected is very close to the number collected for uniform distribution.

The FREAK and SURF descriptors are both scale and rotation invariant. For the performance indicators, it is important to note that both FAST and SURF are both a part of OpenCV and the uniform distribution and HoG features were developed independently specifically for this project.

The results for the different descriptors are shown in table 6.2.

\begin{tabular}{|c|c|c|}
\hline & Two-Class Accuracy & Twenty-Class Accuracy \\
\hline Uniform / HoG & $98.12 \%$ & $64.44 \%$ \\
\hline Fast / FREAK & $84.38 \%$ & $25.31 \%$ \\
\hline SURF & $93.75 \%$ & $39.38 \%$ \\
\hline
\end{tabular}

\begin{tabular}{|c|c|c|}
\hline & Detection Speed & Description Speed \\
\hline Uniform / HoG & $0.037 \mathrm{~ms}$ & $50.98 \mathrm{~ms}$ \\
\hline Fast / FREAK & $0.70 \mathrm{~ms}$ & $66.16 \mathrm{~ms}$ \\
\hline SURF & $104.12 \mathrm{~ms}$ & $138.67 \mathrm{~ms}$ \\
\hline
\end{tabular}

Table 6.2: Feature Detection and Description Performance Comparison

For two-classes, the uniformly distributed features with Histogram of Gradients descriptors miscategorized only three images and significantly outperformed the other two techniques. This might be surprising because many of the images 
in each category are similar to either flipped or rotated versions of one-another, and affine-invariant features would account for that. One possible reason for the lower level of accuracy is that FAST and SURF are both designed for images with more intricate details at a lower pixel level. Additionally, the parameters for the HoG tests were highly tuned for the test set whereas tuning the parameters for the other two detection and description schemes was outside the scope of this project.

\subsection{Bag-of-Features}

The accuracy of the classification depends heavily on how well the bag-offeatures represents the image. The accuracy of the representation is contingent primarily on the size of the visual vocabulary, but also on the way the feature vector is constructed.

This section analyzes the accuracy and speed of several different bag-offeatures representation construction schemes using the feature detection algo-

rithms outlined previously. For classification, a linear SVM is used. In the following test, the speed measures the average time required to calculate the bag-of-features representation for a single image provided the descriptors for that image and a visual-vocabulary.

\subsubsection{Hard Kernel}

Hard kernel assignment simply increments the visual word count in the histogram for the closest visual word centroid in the visual vocabulary. Three different visual vocabulary sizes are used for these tests, 300 visual words, 500 visual 
words, and 800 visual words.

\begin{tabular}{|c|c|c|c|}
\hline$|\mathbf{S}|$ & Two-class Accuracy & Twenty-class Accuracy & BoF Construction Speed \\
\hline \hline 300 & $56.88 \%$ & $8.62 \%$ & $202.48 \mathrm{~ms}$ \\
\hline 500 & $59.38 \%$ & $10.88 \%$ & $320.15 \mathrm{~ms}$ \\
\hline 800 & $43.12 \%$ & $4.00 \%$ & $524.68 \mathrm{~ms}$ \\
\hline
\end{tabular}

Table 6.3: Hard Kernel Classification Performance

The accuracy of the hard-kernel classifier is very, very low. In the case with

the visual vocabulary size of 800 , it under-performs the expected accuracy of random classification (which would be $50 \%$ for the two-class and $5 \%$ for the twenty-class tests.) The tests with the other two visual vocabulary sizes only slightly out-perform random classification.

Regarding the construction speed of the feature vector, no spatial data structure was used to optimize the nearest neighbor search. The running time could be improved by using a spatial data structure to perform the visual-word assignment.

\subsubsection{Soft Kernel}

Soft kernel assignment is used to mitigate the ambiguity of visual word assignment when descriptors fell on visual word boundaries. Each descriptor can contribute to multiple visual words depending on its proximity to each word centroid. As before, three different visual vocabulary sizes are used for these tests, 300 visual words, 500 visual words, and 800 visual words.

The results for soft feature assignment are much better than those for hard feature assignment. Additionally, the accuracy is well above the expected accuracy for random classification. 


\begin{tabular}{|c|c|c|c|}
\hline$|\mathbf{S}|$ & Two-class Accuracy & Twenty-class Accuracy & BoF Construction Speed \\
\hline \hline 300 & $94.38 \%$ & $58.81 \%$ & $267.53 \mathrm{~ms}$ \\
\hline 500 & $94.38 \%$ & $61.31 \%$ & $444.32 \mathrm{~ms}$ \\
\hline 800 & $95.62 \%$ & $61.00 \%$ & $711.84 \mathrm{~ms}$ \\
\hline
\end{tabular}

Table 6.4: Soft Kernel Classification Performance

For the speed tests, the CPU implementation of soft feature assignment is used. No spatial data structures were used for optimization, but it is possible that run-time could be improved by using one.

\subsubsection{Spatial Pyramid}

The spatial pyramid representation is used to encode information about visual word location in the bag-of-features representation. For these tests, soft visual word assignment is used. As in the other two tests, the three different visual vocabulary sizes of 300,500 , and 800 are used, however, the feature vectors are five times larger with sizes of 1500, 2500, and 4000 respectively.

\begin{tabular}{|c|c|c|c|}
\hline$|\mathbf{S}|$ & Two-class Accuracy & Twenty-class Accuracy & BoF Construction Speed \\
\hline \hline 300 & $96.25 \%$ & $61.56 \%$ & $338.19 \mathrm{~ms}$ \\
\hline 500 & $96.88 \%$ & $62.75 \%$ & $561.06 \mathrm{~ms}$ \\
\hline 800 & $96.25 \%$ & $63.94 \%$ & $897.76 \mathrm{~ms}$ \\
\hline
\end{tabular}

Table 6.5: Spatial Pyramid Classification Performance

The spatial pyramid representation enables slightly improved classification performance over only the soft feature assignment. Additionally, the run-time for bag-of-features construction is not impacted too drastically. One downside to the bag-of-features representation is that classifier training was much slower due to the increased dimensionality of the feature-vectors. 


\subsection{Classification}

Three different classification schemes are tested. First, results for the SVM with the linear Kernel are shown again. Second, a Gaussian RBF kernel is used for SVM classification (with SVM paremeters tuned for the highest accuracy). Third, as a baseline, a K-Nearest-Neighbors classifier is tested with $k=10$.

\begin{tabular}{|c|c|c|c|}
\hline$|\mathbf{S}|$ & Two-class Accuracy & Twenty-class Accuracy & Predict Speed (20 class) \\
\hline \hline SVM (Linear) & $98.12 \%$ & $64.44 \%$ & $6.74 \mathrm{~ms}$ \\
\hline SVM (RBF) & $99.38 \%$ & $67.00 \%$ & $11.12 \mathrm{~ms}$ \\
\hline KNN $(K=10)$ & $96.88 \%$ & $56.62 \%$ & $11.68 \mathrm{~ms}$ \\
\hline
\end{tabular}

Table 6.6: Classification Performance

\subsection{Standard Parameters}

For all of the tests performed, the most consistently accurate classification was performed using uniform distribution of features with HoG descriptors. Additionally, a bag-of-features representation constructed with a soft-kernel and a spatial pyramid representation provided higher levels of accuracy. Lastly, SVMs with a Gaussian kernel performed most accurately.

With these in place, several parameters were manipulated to find the maximum possible accuracy with this approach:

- The number uniform features

- The number of spatial bins

- The number of directional bins

- The visual vocabulary size 
- Several SVM specific parameters

To find the maximum accuracy, simulated annealing was used to search the parameter space, where a random neighbor for a parameter set is obtained by multiplying each parameter by a log-normal distributed random number with normal mean 0 and standard deviation 0.1 .

The optimal parameters for the 20-class image set, which yielded the $67 \%$ accuracy, were:

- Number of features: 900

- Direction Bins: 4

- Spatial Bins: 4

- Patch Width: 76

- Visual Vocab Size: 740

- SVM C: 9.0

- SVM Gamma: 0.4

- Spatial Pyramid Depth: 2

Running these parameters on the entire image set yielded $52 \%$ accuracy. 


\section{Chapter 7}

\section{Conclusion}

Much work is still required in the area of computational sketch recognition. The techniques evaluated in this project, although many of them very impressive, are still not accurate enough for practical applications. There are several issues with these approaches and there are also many possible approaches that have not yet been applied to sketch-recognition.

\subsection{Limitations}

The approach studied by this project classifies simple line-drawings that contain a single object in a generally upright orientation. The classifier has no knowledge of the real-world object being represented by the sketch. In order to classify a sketch, human-created drawings are required to train the classifier. This means in order to identify a work of art, there would need to be several instances of similar works of art that already existed and had also been used to train the classifier. 
There were several problems encountered when searching the parameter space for an optimal set of parameters. First, in general, the accuracy of the classification was highly sensitive to small changes in the parameters. This made it difficult to guess optimal parameters when it would have been impractical to search the entire parameter space. Second, due to the OpenCV k-means implementation used, the resulting accuracy of the classification was stochastic, and depending on the test, results could vary up to $\pm 3 \%$. Due to this, an average of two runs was used to find the accuracy when identifying the optimal parameters. However, when searching for parameters several algorithms are better suited for this type of problem than simulated annealing, such as the BOBYQA algorithm $[33]$.

\subsection{Future Work}

There are many performance improvements that could be made that were not evaluated in this project. For example, an optimal parameter search was only performed on the uniform features and the HoG-based descriptors but could have been performed on the SURF features or the FAST/FREAK features and descriptors (and might have achieved a higher level of accuracy.) Several classifiers were not tested which outperform SVMs in certain circumstances like neural networks, boosted decision trees, and others. K-nearest neighbors using a metric like Pearson distance might make more sense given the nature of the classification.

Other projects demonstrate that multiple feature types can be used in conjunction to achieve higher levels of accuracy [22], but this project did not explore this. Applying dimensionality reduction to this problem could potentially provide more information about the data. Using Singular Value Decomposition and La- 
tent Semantic Indexing could be used to describe higher-level features in images using the lower level features.

Other than changing the type of bag-of-features performed, other possibilities could be investigated, for example, generating a hierarchy of sketch classes that are semantically significant and identifying features that enable classification into the hierarchy. An extension of this idea would be to use feature detection to identify sub-pieces of a sketch in conjunction with an ontology engine like OpenCyc to identify objects based on their individual components.

Outside of sketch recognition, results from this project could be used to potentially improve other types of image recognition, or could be used to improve sketch assistance and sketch synthesis. 


\section{Chapter 8}

\section{Bibliography}

[1] Alexandre Alahi, Raphael Ortiz, and Pierre Vandergheynst. Freak: Fast retina keypoint. In Computer Vision and Pattern Recognition (CVPR), 2012 IEEE Conference on, pages 510-517. IEEE, 2012.

[2] Herbert Bay, Tinne Tuytelaars, and Luc Van Gool. Surf: Speeded up robust features. In Computer Vision-ECCV 2006, pages 404-417. Springer, 2006.

[3] Serge Belongie, Jitendra Malik, and Jan Puzicha. Shape matching and object recognition using shape contexts. Pattern Analysis and Machine Intelligence, IEEE Transactions on, 24(4):509-522, 2002.

[4] C. J. C. Burges. A tutorial on support vector machines for pattern recognition. Data Mining and Knowledge Discovery, 2(2):1-47, 1998.

[5] John Canny. A computational approach to edge detection. Pattern Analysis and Machine Intelligence, IEEE Transactions on, (6):679-698, 1986.

[6] Abdolah Chalechale, Golshah Naghdy, and Alfred Mertins. Sketch-based 
image matching using angular partitioning. Systems, Man and Cybernetics, Part A: Systems and Humans, IEEE Transactions on, 35(1):28-41, 2005.

[7] Tao Chen, Ming-Ming Cheng, Ping Tan, Ariel Shamir, and Shi-Min Hu. Sketch2photo: internet image montage. In ACM Transactions on Graphics (TOG), volume 28, page 124. ACM, 2009.

[8] Gabriella Csurka, Christopher Dance, Lixin Fan, Jutta Willamowski, and Cédric Bray. Visual categorization with bags of keypoints. In Workshop on statistical learning in computer vision, ECCV, volume 1, page 22, 2004.

[9] Navneet Dalal and Bill Triggs. Histograms of oriented gradients for human detection. In Computer Vision and Pattern Recognition, 2005. CVPR 2005. IEEE Computer Society Conference on, volume 1, pages 886-893. IEEE, 2005.

[10] Daniel Dixon, Manoj Prasad, and Tracy Hammond. icandraw: using sketch recognition and corrective feedback to assist a user in drawing human faces. In Proceedings of the 28th international conference on Human factors in computing systems, pages 897-906. ACM, 2010.

[11] Mathias Eitz, Kristian Hildebrand, Tamy Boubekeur, and Marc Alexa. Sketch-based image retrieval: Benchmark and bag-of-features descriptors. Visualization and Computer Graphics, IEEE Transactions on, 17(11):16241636, 2011.

[12] Mathias Eitz, James Hays, and Marc Alexa. How do humans sketch objects? ACM Transactions on Graphics (Proceedings SIGGRAPH), 31(4): 44:1-44:10, 2012. 
[13] Li Fei-Fei and Pietro Perona. A bayesian hierarchical model for learning natural scene categories. In Computer Vision and Pattern Recognition, 2005. CVPR 2005. IEEE Computer Society Conference on, volume 2, pages 524531. IEEE, 2005.

[14] Wolfgang Förstner and Eberhard Gülch. A fast operator for detection and precise location of distinct points, corners and centres of circular features. In Proc. ISPRS intercommission conference on fast processing of photogrammetric data, pages 281-305, 1987.

[15] Tracy Hammond and Randall Davis. Ladder, a sketching language for user interface developers. Computers $\& 3$ Graphics, 29(4):518-532, 2005.

[16] Chris Harris and Mike Stephens. A combined corner and edge detector. In Alvey vision conference, volume 15, page 50. Manchester, UK, 1988.

[17] Peter Henry, Michael Krainin, Evan Herbst, Xiaofeng Ren, and Dieter Fox. Rgb-d mapping: Using depth cameras for dense 3d modeling of indoor environments. In the 12th International Symposium on Experimental Robotics (ISER), volume 20, pages 22-25, 2010.

[18] Anil K Jain, Salil Prabhakar, Lin Hong, and Sharath Pankanti. Filterbankbased fingerprint matching. Image Processing, IEEE Transactions on, 9(5): 846-859, 2000.

[19] Yu-Gang Jiang, Chong-Wah Ngo, and Jun Yang. Towards optimal bag-offeatures for object categorization and semantic video retrieval. In Proceedings of the 6th ACM international conference on Image and video retrieval, pages 494-501. ACM, 2007. 
[20] James A Landay and Brad A Myers. Sketching interfaces: Toward more human interface design. Computer, 34(3):56-64, 2001.

[21] Joseph J LaViola Jr and Robert C Zeleznik. Mathpad 2: a system for the creation and exploration of mathematical sketches. In ACM SIGGRAPH 2007 courses, page 46. ACM, 2007.

[22] Svetlana Lazebnik, Cordelia Schmid, and Jean Ponce. Beyond bags of features: Spatial pyramid matching for recognizing natural scene categories. In Computer Vision and Pattern Recognition, 2006 IEEE Computer Society Conference on, volume 2, pages 2169-2178. IEEE, 2006.

[23] Yong Jae Lee, C Lawrence Zitnick, and Michael F Cohen. Shadowdraw: realtime user guidance for freehand drawing. In ACM Transactions on Graphics (TOG), volume 30, page 27. ACM, 2011.

[24] David G Lowe. Object recognition from local scale-invariant features. In Computer vision, 1999. The proceedings of the seventh IEEE international conference on, volume 2, pages 1150-1157. Ieee, 1999.

[25] David Marr and Ellen Hildreth. Theory of edge detection. Proceedings of the Royal Society of London. Series B. Biological Sciences, 207(1167):187-217, 1980.

[26] Krystian Mikolajczyk, Tinne Tuytelaars, Cordelia Schmid, Andrew Zisserman, Jiri Matas, Frederik Schaffalitzky, Timor Kadir, and Luc Van Gool. A comparison of affine region detectors. International journal of computer vision, 65(1-2):43-72, 2005.

[27] Kai Nickel and Rainer Stiefelhagen. Visual recognition of pointing gestures 
for human-robot interaction. Image and Vision Computing, 25(12):1875$1884,2007$.

[28] Giuseppe Oriolo, Giovanni Ulivi, and Marilena Vendittelli. Real-time map building and navigation for autonomous robots in unknown environments. Systems, Man, and Cybernetics, Part B: Cybernetics, IEEE Transactions on, 28(3):316-333, 1998.

[29] Tom Y Ouyang and Randall Davis. Chemink: a natural real-time recognition system for chemical drawings. In Proceedings of the 16th international conference on Intelligent user interfaces, pages 267-276. ACM, 2011.

[30] Brandon Paulson and Tracy Hammond. Paleosketch: accurate primitive sketch recognition and beautification. In Proceedings of the 13th international conference on Intelligent user interfaces, pages 1-10. ACM, 2008.

[31] Pietro Perona and Jitendra Malik. Scale-space and edge detection using anisotropic diffusion. Pattern Analysis and Machine Intelligence, IEEE Transactions on, 12(7):629-639, 1990.

[32] James Philbin, Ondrej Chum, Michael Isard, Josef Sivic, and Andrew Zisserman. Lost in quantization: Improving particular object retrieval in large scale image databases. In Computer Vision and Pattern Recognition, 2008. CVPR 2008. IEEE Conference on, pages 1-8. IEEE, 2008.

[33] Michael JD Powell. The bobyqa algorithm for bound constrained optimization without derivatives. Cambridge NA Report NA2009/06, University of Cambridge, Cambridge, 2009.

[34] Edward Rosten and Tom Drummond. Machine learning for high-speed cor- 
ner detection. In Computer Vision-ECCV 2006, pages 430-443. Springer, 2006.

[35] Dean Rubine. Specifying gestures by example, volume 25. ACM, 1991.

[36] Ethan Rublee, Vincent Rabaud, Kurt Konolige, and Gary Bradski. Orb: an efficient alternative to sift or surf. In Computer Vision (ICCV), 2011 IEEE International Conference on, pages 2564-2571. IEEE, 2011.

[37] Gerard Salton and Michael J McGill. Introduction to modern information retrieval. McGraw-Hill, Inc., 1986.

[38] Oliver G Selfridge. Pattern recognition and modern computers. In Proceedings of the March 1-3, 1955, western joint computer conference, pages 91-93. ACM, 1955.

[39] Taku Senoo, Akio Namiki, and Masatoshi Ishikawa. High-speed batting using a multi-jointed manipulator. In Robotics and Automation, 2004. Proceedings. ICRA'04. 2004 IEEE International Conference on, volume 2, pages 11911196. IEEE, 2004.

[40] Tevfik Metin Sezgin, Thomas Stahovich, and Randall Davis. Sketch based interfaces: early processing for sketch understanding. In $A C M S I G G R A P H$ 2006 Courses, page 22. ACM, 2006.

[41] Abhinav Shrivastava, Tomasz Malisiewicz, Abhinav Gupta, and Alexei A Efros. Data-driven visual similarity for cross-domain image matching. ACM Transactions on Graphics (TOG), 30(6), 2011.

[42] Josef Sivic and Andrew Zisserman. Video google: A text retrieval approach to object matching in videos. In Computer Vision, 2003. Proceedings. Ninth IEEE International Conference on, pages 1470-1477. IEEE, 2003. 
[43] Matthew Turk and Alex Pentland. Eigenfaces for recognition. Journal of cognitive neuroscience, 3(1):71-86, 1991.

[44] V. Vapnik. The Nature of Statistical Learning Theory. Springer-Verlag, New York, 1995.

[45] Paul Viola and Michael Jones. Rapid object detection using a boosted cascade of simple features. In Computer Vision and Pattern Recognition, 2001. CVPR 2001. Proceedings of the 2001 IEEE Computer Society Conference on, volume 1, pages I-511. IEEE, 2001.

[46] C Lawrence Zitnick. Binary coherent edge descriptors. In Computer VisionECCV 2010, pages 170-182. Springer, 2010. 